\title{
INTERPOLATION BETWEEN HILBERT SPACES
}

\author{
YACIN AMEUR
}

\begin{abstract}
This note comprises a synthesis of certain results in the theory of exact interpolation between Hilbert spaces. In particular, we examine various characterizations of interpolation spaces and their relations to a number of results in operator-theory and in function-theory.
\end{abstract}

\section{INTERPOLATION THEORETIC NOTIONS}

1.1. Interpolation norms. When $X, Y$ are normed spaces, we use the symbol $\mathcal{L}(X ; Y)$ to denote the totality of bounded linear maps $T: X \rightarrow Y$ with the operator norm

$$
\|T\|_{\mathcal{L}(X ; Y)}=\sup \left\{\|T x\|_{Y} ;\|x\|_{X} \leq 1\right\} .
$$

When $X=Y$ we simply write $\mathcal{L}(X)$.

Consider a pair of Hilbert spaces $\overline{\mathcal{H}}=\left(\mathcal{H}_{0}, \mathcal{H}_{1}\right)$ which is regular in the sense that $\mathcal{H}_{0} \cap \mathcal{H}_{1}$ is dense in $\mathcal{H}_{0}$ as well as in $\mathcal{H}_{1}$. We assume that the pair is compatible, i.e., both $\mathcal{H}_{i}$ are embedded in some common Hausdorff topological vector space $\mathcal{M}$.

We define the $K$-functional (1) for the couple $\overline{\mathcal{H}}$ by

$$
K(t, x)=K(t, x ; \overline{\mathcal{H}})=\inf _{x=x_{0}+x_{1}}\left\{\left\|x_{0}\right\|_{0}^{2}+t\left\|x_{1}\right\|_{1}^{2}\right\}, \quad t>0, x \in \mathcal{M} .
$$

The sum of the spaces $\mathcal{H}_{0}$ and $\mathcal{H}_{1}$ is defined to be the space consisting of all $x \in \mathcal{M}$ such that the quantity $\|x\|_{\Sigma}^{2}:=K(1, x)$ is finite; we denote this space by the symbols

$$
\Sigma=\Sigma(\overline{\mathcal{H}})=\mathcal{H}_{0}+\mathcal{H}_{1} .
$$

We shall soon see that $\Sigma$ is a Hilbert space (see Lemma 1.1). The intersection

$$
\Delta=\Delta(\overline{\mathcal{H}})=\mathcal{H}_{0} \cap \mathcal{H}_{1}
$$

is a Hilbert space under the norm $\|x\|_{\Delta}^{2}:=\|x\|_{0}^{2}+\|x\|_{1}^{2}$.

A map $T: \Sigma(\overline{\mathcal{H}}) \rightarrow \Sigma(\overline{\mathcal{K}})$ is called a couple map from $\overline{\mathcal{H}}$ to $\overline{\mathcal{K}}$ if the restriction of $T$ to $\mathcal{H}_{i}$ maps $\mathcal{H}_{i}$ boundedly into $\mathcal{K}_{i}$ for $i=0,1$. We use the notations $T \in \mathcal{L}(\overline{\mathcal{H}} ; \overline{\mathcal{K}})$ or $T: \overline{\mathcal{H}} \rightarrow \overline{\mathcal{K}}$ to denote that $T$ is a couple map. It is easy to check that $\mathcal{L}(\overline{\mathcal{H}} ; \overline{\mathcal{K}})$ is a Banach space, when equipped with the norm

$$
\|T\|_{\mathcal{L}(\overline{\mathcal{H}} ; \overline{\mathcal{K}})}:=\max _{j=0,1}\left\{\|T\|_{\mathcal{L}\left(\mathcal{H}_{j} ; \mathcal{K}_{j}\right)}\right\} .
$$

If $\|T\|_{\mathcal{L}(\overline{\mathcal{H}} ; \overline{\mathcal{K}})} \leq 1$ we speak of a contraction from $\overline{\mathcal{H}}$ to $\overline{\mathcal{K}}$.

A Banach space $X$ such that $\Delta \subset X \subset \Sigma$ (continuous inclusions) is called intermediate with respect to the pair $\overline{\mathcal{H}}$.

\footnotetext{
${ }^{1}$ More precisely, this is the quadratic version of the classical Peetre $K$-functional.
} 
Let $X, Y$ be intermediate spaces with respect to couples $\overline{\mathcal{H}}, \overline{\mathcal{K}}$, respectively. We say that $X, Y$ are (relative) interpolation spaces if there is a constant $C$ such that $T: \overline{\mathcal{H}} \rightarrow \overline{\mathcal{K}}$ implies that $T: X \rightarrow Y$ and

$$
\|T\|_{\mathcal{L}(X ; Y)} \leq C\|T\|_{\mathcal{L}(\overline{\mathcal{H}} ; \overline{\mathcal{K}})} .
$$

In the case when $C=1$ we speak about exact interpolation. When $\overline{\mathcal{H}}=\overline{\mathcal{K}}$ and $X=Y$ we simply say that $X$ is an (exact) interpolation space with respect to $\overline{\mathcal{H}}$.

Let $H$ be a suitable function of two positive variables and $X, Y$ spaces intermediate to the couples $\overline{\mathcal{H}}, \overline{\mathcal{K}}$, respectively. We say that the spaces $X, Y$ are of type $H$ (relative to $\overline{\mathcal{H}}, \overline{\mathcal{K}}$ ) if for any positive numbers $M_{0}, M_{1}$ we have

$$
\|T\|_{\mathcal{L}\left(\mathcal{H}_{i} ; \mathcal{K}_{i}\right)} \leq M_{i}, \quad i=0,1 \quad \text { implies } \quad\|T\|_{\mathcal{L}(X ; Y)} \leq H\left(M_{0}, M_{1}\right) .
$$

The case $H(x, y)=\max \{x, y\}$ corresponds to exact interpolation, while $H(x, y)=$ $x^{1-\theta} y^{\theta}$ corresponds to the convexity estimate

$$
\|T\|_{\mathcal{L}(X ; Y)} \leq\|T\|_{\mathcal{L}\left(\mathcal{H}_{0} ; \mathcal{K}_{0}\right)}^{1-\theta}\|T\|_{\mathcal{L}\left(\mathcal{H}_{1} ; \mathcal{K}_{1}\right)}^{\theta} .
$$

In the situation of (1.4), one says that the interpolation spaces $X, Y$ are of exponent $\theta$ with respect to the pairs $\overline{\mathcal{H}}, \overline{\mathcal{K}}$.

1.2. $K$-spaces. Given a regular Hilbert couple $\overline{\mathcal{H}}$ and a positive Radon measure $\varrho$ on the compactified half-line $[0, \infty]$ we define an intermediate quadratic norm by

$$
\|x\|_{*}^{2}=\|x\|_{\varrho}^{2}=\int_{[0, \infty]}\left(1+t^{-1}\right) K(t, x ; \overline{\mathcal{H}}) d \varrho(t) .
$$

Here the integrand $k(t)=\left(1+t^{-1}\right) K(t, x)$ is defined at the points 0 and $\infty$ by $k(0)=\|x\|_{1}^{2}$ and $k(\infty)=\|x\|_{0}^{2}$; we shall write $\mathcal{H}_{*}$ or $\mathcal{H}_{\varrho}$ for the Hilbert space defined by the norm (1.5).

Let $T \in \mathcal{L}(\overline{\mathcal{H}} ; \overline{\mathcal{K}})$ and suppose that $\|T\|_{\mathcal{L}\left(\mathcal{H}_{i} ; \mathcal{K}_{i}\right)} \leq M_{i}$; then

$$
K(t, T x ; \overline{\mathcal{K}}) \leq M_{0}^{2} K\left(M_{1}^{2} t / M_{0}^{2}, x ; \overline{\mathcal{H}}\right), \quad x \in \Sigma .
$$

In particular, $M_{i} \leq 1$ for $i=0,1$ implies $\|T x\|_{\mathcal{K}_{\varrho}} \leq\|x\|_{\mathcal{H}_{\varrho}}$ for all $x \in \mathcal{H}_{\varrho}$. It follows that the spaces $\mathcal{H}_{\varrho}, \mathcal{K}_{\varrho}$ are exact interpolation spaces with respect to $\overline{\mathcal{H}}, \overline{\mathcal{K}}$.

Geometric interpolation. When the measure $\varrho$ is given by

$$
d \varrho(t)=c_{\theta} \frac{t^{-\theta}}{1+t} d t, \quad c_{\theta}=\frac{\pi}{\sin \theta \pi}, \quad 0<\theta<1,
$$

we denote the norm (1.5) by

$$
\|x\|_{\theta}^{2}:=c_{\theta} \int_{0}^{\infty} t^{-\theta} K(t, x) \frac{d t}{t} .
$$

The corresponding space $\mathcal{H}_{\theta}$ is easily seen to be of exponent $\theta$ with respect to $\overline{\mathcal{H}}$. In 3.1] we will recognize $\mathcal{H}_{\theta}$ as the geometric interpolation space which has been studied independently by several authors, see [27, 40, 25]. 
1.3. Pick functions. Let $\overline{\mathcal{H}}$ be a regular Hilbert couple. The squared norm $\|x\|_{1}^{2}$ is a densely defined quadratic form in $\mathcal{H}_{0}$, which we represent as

$$
\|x\|_{1}^{2}=\langle A x, x\rangle_{0}=\left\|A^{1 / 2} x\right\|_{0}^{2}
$$

where $A$ is a densely defined, positive, injective (perhaps unbounded) operator in $\mathcal{H}_{0}$. The domain of the positive square-root $A^{1 / 2}$ is $\Delta$.

Lemma 1.1. We have in terms of the functional calculus in $\mathcal{H}_{0}$

$$
K(t, x)=\left\langle\frac{t A}{1+t A} x, x\right\rangle_{0}, \quad t>0 .
$$

In the formula (1.8), we have identified the bounded operator $\frac{t A}{1+t A}$ with its extension to $\mathcal{H}_{0}$.

Proof. Fix $x \in \Delta$. By a straightforward convexity argument, there is a unique decomposition $x=x_{0, t}+x_{1, t}$ which is optimal in the sense that

$$
K(t, x)=\left\|x_{0, t}\right\|_{0}^{2}+t\left\|x_{1, t}\right\|_{1}^{2} .
$$

It follows that $x_{i, t} \in \Delta$ for $i=0,1$. Moreover, for all $y \in \Delta$ we have

$$
\left.\frac{d}{d \epsilon}\left\{\left\|x_{0, t}+\epsilon y\right\|_{0}^{2}+t\left\|x_{1, t}-\epsilon y\right\|_{1}^{2}\right\}\right|_{\epsilon=0}=0
$$

i.e.,

$$
\left\langle A^{-1 / 2} x_{0, t}-t A^{1 / 2} x_{1, t}, A^{1 / 2} y\right\rangle_{0}=0, \quad y \in \Delta .
$$

By regularity, we conclude that $A^{-1 / 2} x_{0, t}=t A^{1 / 2} x_{1, t}$, whence

$$
x_{0, t}=\frac{t A}{1+t A} x \quad \text { and } \quad x_{1, t}=\frac{1}{1+t A} x .
$$

(Note that the operators in (1.10) extend to bounded operators on $\mathcal{H}_{0}$.) Inserting the relations (1.10) into (1.9), one finishes the proof of the lemma.

Now fix a positive Radon measure $\varrho$ on $[0, \infty]$. The norm in the space $\mathcal{H}_{\varrho}$ (see (1.5)) can be written

$$
\|x\|_{\varrho}^{2}=\langle h(A) x, x\rangle_{0},
$$

where

$$
h(\lambda)=\int_{[0, \infty]} \frac{(1+t) \lambda}{1+t \lambda} d \varrho(t) .
$$

The class of functions representable in this form for some positive Radon measure $\varrho$ is the class $P^{\prime}$ of Pick functions, positive and regular on $\mathbf{R}_{+}$.

Notice that for the definition (1.11) to make sense, we just need $h$ to be defined on $\sigma(A) \backslash\{0\}$, where $\sigma(A)$ is the spectrum of $A$. (The value $h(0)$ is irrelevant since $A$ is injective).

A calculus exercise shows that for the space $\mathcal{H}_{\theta}$ (see (1.7)) we have

$$
\|x\|_{\theta}^{2}=\left\langle A^{\theta} x, x\right\rangle_{0} .
$$


1.4. Quadratic interpolation norms. Let $\mathcal{H}_{*}$ be any quadratic intermediate space relative to $\overline{\mathcal{H}}$. We write

$$
\|x\|_{*}^{2}=\langle B x, x\rangle_{0}
$$

where $B$ is a positive injective operator in $\mathcal{H}_{0}$ (the domain of $B^{1 / 2}$ is $\Delta$ ).

For a map $T \in \mathcal{L}(\overline{\mathcal{H}})$ we shall often use the simplified notations

$$
\|T\|=\|T\|_{\mathcal{L}\left(\mathcal{H}_{0}\right)} \quad, \quad\|T\|_{A}=\|T\|_{\mathcal{L}\left(\mathcal{H}_{1}\right)} \quad, \quad\|T\|_{B}=\|T\|_{\mathcal{L}\left(\mathcal{H}_{*}\right)} .
$$

The reader can check the identities

$$
\|T\|_{A}=\left\|A^{1 / 2} T A^{-1 / 2}\right\| \quad \text { and } \quad\|T\|_{B}=\left\|B^{1 / 2} T B^{-1 / 2}\right\| .
$$

We shall refer to the following lemma as Donoghue's lemma, cf. [14, Lemma 1].

Lemma 1.2. If $\mathcal{H}_{*}$ is exact interpolation with respect to $\overline{\mathcal{H}}$, then $B$ commutes with every projection which commutes with $A$ and $B=h(A)$ where $h$ is some positive Borel function on $\sigma(A)$.

Proof. For an orthogonal projection $E$ on $\mathcal{H}_{0}$, the condition $\|E\|_{A} \leq 1$ is equivalent to that $E A E \leq A$, i.e., that $E$ commutes with $A$. The hypothesis that $\mathcal{H}_{*}$ be exact interpolation thus implies that every spectral projection of $A$ commutes with $B$. It now follows from von Neumann's bicommutator theorem that $B=h(A)$ for some positive Borel function $h$ on $\sigma(A)$.

In view of the lemma, the characterization of the exact quadratic interpolation norms of a given type $H$ reduces to the characterization of functions $h: \sigma(A) \rightarrow \mathbf{R}_{+}$ such that for all $T \in \mathcal{L}(\overline{\mathcal{H}})$

$$
\|T\| \leq M_{0} \quad \text { and } \quad\|T\|_{A} \leq M_{1} \quad \Rightarrow \quad\|T\|_{h(A)} \leq H\left(M_{0}, M_{1}\right),
$$

or alternatively,

$$
T^{*} T \leq M_{0}^{2} \quad \text { and } \quad T^{*} A T \leq M_{1}^{2} A \quad \Rightarrow \quad T^{*} h(A) T \leq H\left(M_{0}, M_{1}\right)^{2} h(A) .
$$

The set of functions $h: \sigma(A) \rightarrow \mathbf{R}_{+}$satisfying these equivalent conditions forms a convex cone $C_{H, A}$; its elements are called interpolation functions of type $H$ relative to $A$. In the case when $H(x, y)=\max \{x, y\}$ we simply write $C_{A}$ for $C_{H, A}$ and speak of exact interpolation functions relative to $A$.

1.5. Exact Calderón pairs and the $K$-property. Given two intermediate normed spaces $Y, X$ relative to $\overline{\mathcal{H}}, \overline{\mathcal{K}}$, we say that they are (relatively) exact $K$-monotonic if the conditions

$$
x^{0} \in X \quad \text { and } \quad K\left(t, y^{0} ; \overline{\mathcal{H}}\right) \leq K\left(t, x^{0} ; \overline{\mathcal{K}}\right), \quad t>0
$$

imply that

$$
y^{0} \in Y \quad \text { and } \quad\left\|y^{0}\right\|_{Y} \leq\left\|x^{0}\right\|_{X}
$$

It is easy to see that exact $K$-monotonicity implies exact interpolation.

Proof of this. If $\|T\|_{\mathcal{L}(\overline{\mathcal{K}} ; \overline{\mathcal{H}})} \leq 1$ then $\forall x, t: K(t, T x ; \overline{\mathcal{H}}) \leq K(t, x ; \overline{\mathcal{K}})$ whence $\|T x\|_{Y} \leq\|x\|_{X}$, by exact $K$-monotonicity. Hence $\|T\|_{\mathcal{L}(X ; Y)} \leq 1$. 
Two pairs $\overline{\mathcal{H}}, \overline{\mathcal{K}}$ are called exact relative Calderón pairs if any two exact interpolation (Banach-) spaces $Y, X$ are exact $K$-monotonic. Thus, with respect to to exact Calderón pairs, exact interpolation is equivalent to exact $K$-monotonicity. The term "Calderón pair" was coined after thorough investigation of A. P. Calderón's study of the pair $\left(L_{1}, L_{\infty}\right)$, see [10] and [11.

In our present discussion, it is not convenient to work directly with the definition of exact Calderón pairs. Instead, we shall use the following, closely related notion.

We say that a pair of couples $\overline{\mathcal{H}}, \overline{\mathcal{K}}$ has the relative (exact) $K$-property if for all $x^{0} \in \Sigma(\overline{\mathcal{K}})$ and $y^{0} \in \Sigma(\overline{\mathcal{H}})$ such that

$$
K\left(t, y^{0} ; \overline{\mathcal{H}}\right) \leq K\left(t, x^{0} ; \overline{\mathcal{K}}\right), \quad t>0,
$$

there exists a map $T \in \mathcal{L}(\overline{\mathcal{K}} ; \overline{\mathcal{H}})$ such that $T x^{0}=y^{0}$ and $\|T\|_{\mathcal{L}(\overline{\mathcal{K}} ; \overline{\mathcal{H}})} \leq 1$.

Lemma 1.3. If $\overline{\mathcal{H}}, \overline{\mathcal{K}}$ have the relative $K$-property, then they are exact relative Calderón pairs.

Proof. Let $Y, X$ be exact interpolation spaces relative to $\overline{\mathcal{H}}, \overline{\mathcal{K}}$ and take $x^{0} \in X$ and $y^{0} \in \Sigma(\overline{\mathcal{H}})$ such that (1.16) holds. By the $K$-property there is $T: \overline{\mathcal{K}} \rightarrow \overline{\mathcal{H}}$ such that $T x^{0}=y^{0}$ and $\|T\| \leq 1$. Then $\|T\|_{\mathcal{L}(X ; Y)} \leq 1$, and so $\left\|y^{0}\right\|_{Y}=\left\|T x^{0}\right\|_{Y} \leq$ $\left\|x^{0}\right\|_{X}$. We have shown that $Y, X$ are exact $K$-monotonic.

In the diagonal case $\overline{\mathcal{H}}=\overline{\mathcal{K}}$, we simply say that $\overline{\mathcal{H}}$ is an exact Calderón couple if for intermediate spaces $Y, X$, the property of being exact interpolation is equivalent to being exact $K$-monotonic. Likewise, we say that $\overline{\mathcal{H}}$ has the $K$-property if the pair of couples $\overline{\mathcal{H}}, \overline{\mathcal{H}}$ has that property.

Remark 1.4. For an operator $T: \overline{\mathcal{K}} \rightarrow \overline{\mathcal{H}}$ to be a contraction, it is necessary and sufficient that

$$
K(t, T x ; \overline{\mathcal{H}}) \leq K(t, x ; \overline{\mathcal{K}}), \quad x \in \Sigma(\overline{\mathcal{K}}), t>0
$$

Indeed, the necessity is immediate. To prove the sufficiency it suffices to observe that letting $t \rightarrow \infty$ in (1.17) gives $\|T x\|_{0} \leq\|x\|_{0}$, and dividing (1.17) by $t$, and then letting $t \rightarrow 0$, gives that $\|T x\|_{1} \leq\|x\|_{1}$.

\section{Mapping properties of Hilbert couples}

2.1. Main results. We shall elaborate on the following main result from [2].

Theorem I. Any pair of regular Hilbert couples $\overline{\mathcal{H}}, \overline{\mathcal{K}}$ has the relative $K$-property .

Before we come to the proof of Theorem \, we note some consequences of it. We first have the following corollary, which shows that a strong form of the $K$-property is true.

Corollary 2.1. Let $\overline{\mathcal{H}}$ be a regular Hilbert couple and $x^{0}, y^{0} \in \Sigma$ elements such that

$$
K\left(t, y^{0}\right) \leq M_{0}^{2} K\left(M_{1}^{2} t / M_{0}^{2}, x^{0}\right), \quad t>0 .
$$

(i) There exists a map $T \in \mathcal{L}(\overline{\mathcal{H}})$ such that $T x^{0}=y^{0}$ and $\|T\|_{\mathcal{L}\left(\mathcal{H}_{i}\right)} \leq M_{i}$, $i=0,1$.

(ii) If $x^{0} \in X$ where $X$ is an interpolation space of type $H$, then

$$
\left\|y^{0}\right\|_{X} \leq H\left(M_{0}, M_{1}\right)\left\|x^{0}\right\|_{X}
$$


Proof. (i) Introduce a new couple $\overline{\mathcal{K}}$ by letting $\|x\|_{\mathcal{K}_{i}}=M_{i}\|x\|_{\mathcal{H}_{i}}$. The relation (2.1) then says that

$$
K\left(t, y^{0} ; \overline{\mathcal{H}}\right) \leq K\left(t, x^{0} ; \overline{\mathcal{K}}\right), \quad t>0 .
$$

By Theorem $\prod$ there is a contraction $T: \overline{\mathcal{K}} \rightarrow \overline{\mathcal{H}}$ such that $T x^{0}=y^{0}$. It now suffices to note that $\|T\|_{\mathcal{L}\left(\mathcal{H}_{i}\right)}=M_{i}\|T\|_{\mathcal{L}\left(\mathcal{K}_{i} ; \mathcal{H}_{i}\right)}$; (ii) then follows from Lemma 1.3

We next mention some equivalent versions of Theorem [ which uses the families of functionals $K_{p}$ and $E_{p}$ defined (for $p \geq 1$ and $t, s>0$ ) via

$$
\begin{aligned}
& K_{p}(t)=K_{p}(t, x)=K_{p}(t, x ; \overline{\mathcal{H}})=\inf _{x=x_{0}+x_{1}}\left\{\left\|x_{0}\right\|_{0}^{p}+t\left\|x_{1}\right\|_{1}^{p}\right\} \\
& E_{p}(s)=E_{p}(s, x)=E_{p}(s, x ; \overline{\mathcal{H}})=\inf _{\left\|x_{0}\right\|_{0}^{p} \leq s}\left\{\left\|x-x_{0}\right\|_{1}^{p}\right\} .
\end{aligned}
$$

Note that $K=K_{2}$ and that $E_{p}(s)=E_{1}\left(s^{1 / p}\right)^{p}$; the $E$-functionals are used in approximation theory. One has that $E_{p}$ is decreasing and convex on $\mathbf{R}_{+}$and that

$$
K_{p}(t)=\inf _{s>0}\left\{s+t E_{p}(s)\right\},
$$

which means that $K_{p}$ is a kind of Legendre transform of $E_{p}$. The inverse Legendre transformation takes the form

$$
E_{p}(s)=\sup _{t>0}\left\{\frac{K_{p}(t)}{t}-\frac{s}{t}\right\} .
$$

It is now immediate that, for all $x \in \Sigma(\overline{\mathcal{K}})$ and $y \in \Sigma(\overline{\mathcal{H}})$, we have

$$
K_{p}(t, y) \leq K_{p}(t, x), \quad t>0 \quad \Leftrightarrow \quad E_{p}(s, y) \leq E_{p}(s, x), \quad s>0 .
$$

Since moreover $E_{p}(s)=E_{2}\left(s^{2 / p}\right)^{p / 2}$, the conditions in (2.3) are equivalent to that $K(t, y) \leq K(t, x)$ for all $t>0$. We have shown the following result.

Corollary 2.2. In Theorem $\square$, one can substitute the $K$-functional for any of the functionals $K_{p}$ or $E_{p}$.

Define an exact interpolation norm $\|\cdot\|_{\varrho, p}$ relative to $\overline{\mathcal{H}}$ by

$$
\|x\|_{\varrho, p}^{p}=\int_{[0, \infty]}\left(1+t^{-1}\right) K_{p}(t, x) d \varrho(t)
$$

where $\varrho$ is a positive Radon measure on $[0, \infty]$. This norm is non-quadratic when $p \neq 2$, but is of course equivalent to the quadratic norm corresponding to $p=2$.

2.2. Reduction to the diagonal case. It is not hard to reduce the discussion of Theorem \ to a diagonal situation.

Lemma 2.3. If the $K$-propertyholds for regular Hilbert couples in the diagonal case $\overline{\mathcal{H}}=\overline{\mathcal{K}}$, then it holds in general.

Proof. Fix elements $y^{0} \in \Sigma(\overline{\mathcal{H}})$ and $x^{0} \in \Sigma(\overline{\mathcal{K}})$ such that the inequality (1.16) holds. We must construct a map $T: \overline{\mathcal{K}} \rightarrow \overline{\mathcal{H}}$ such that $T x^{0}=y^{0}$ and $\|T\| \leq 1$.

To do this, we form the direct sum $\overline{\mathcal{S}}=\left(\mathcal{H}_{0} \oplus \mathcal{K}_{0}, \mathcal{H}_{1} \oplus \mathcal{K}_{1}\right)$. It is clear that $\mathcal{S}_{0}+\mathcal{S}_{1}=\left(\mathcal{H}_{0}+\mathcal{H}_{1}\right) \oplus\left(\mathcal{K}_{0}+\mathcal{K}_{1}\right)$, and that

$$
K(t, x \oplus y ; \overline{\mathcal{S}})=K(t, x ; \overline{\mathcal{H}})+K(t, y ; \overline{\mathcal{K}}) .
$$

Then

$$
K\left(t, 0 \oplus y^{0} ; \overline{\mathcal{S}}\right) \leq K\left(t, x^{0} \oplus 0 ; \overline{\mathcal{S}}\right)
$$


Hence assuming that the couple $\overline{\mathcal{S}}$ has the $K$-property, we can assert the existence of a map $S \in \mathcal{L}(\overline{\mathcal{S}})$ such that $S\left(x^{0} \oplus 0\right)=0 \oplus y^{0}$ and $\|S\| \leq 1$. Letting $P$ : $\mathcal{S}_{0}+\mathcal{S}_{1} \rightarrow \mathcal{K}_{0}+\mathcal{K}_{1}$ be the orthogonal projection, the assignment $T x=P S(x \oplus 0)$ now defines a map such that $T x^{0}=y^{0}$ and $\|T\|_{\mathcal{L}(\overline{\mathcal{H}} ; \overline{\mathcal{K}})} \leq 1$.

2.3. The principal case. The core content of Theorem \is contained in the following statement.

Theorem 2.4. Suppose that a regular Hilbert couple $\overline{\mathcal{H}}$ is finite dimensional and that all eigenvalues of the corresponding operator $A$ are of unit multiplicity. Then $\overline{\mathcal{H}}$ has the K-property.

We shall settle for proving Lemma 2.4 in this section, postponing to Section 5 the general case of Theorem [.

To prepare for the proof, we write the eigenvalues $\lambda_{i}$ of $A$ in increasing order,

$$
\sigma(A)=\left\{\lambda_{i}\right\}_{1}^{n} \text { where } 0<\lambda_{1}<\cdots<\lambda_{n} .
$$

Let $e_{i}$ be corresponding eigenvectors of unit length for the norm of $\mathcal{H}_{0}$. Then for a vector $x=\sum x_{i} e_{i}$ we have

$$
\|x\|_{0}^{2}=\sum_{1}^{n}\left|x_{i}\right|^{2} \quad, \quad\|x\|_{1}^{2}=\sum_{1}^{n} \lambda_{i}\left|x_{i}\right|^{2} .
$$

Working in the coordinate system $\left(e_{i}\right)$, the couple $\overline{\mathcal{H}}$ becomes identified with the $n$-dimensional weighted $\ell_{2}$ couple

$$
\overline{\ell_{2}^{n}}(\lambda):=\left(\ell_{2}^{n}, \ell_{2}^{n}(\lambda)\right),
$$

where we write $\lambda$ for the sequence $\left(\lambda_{i}\right)_{1}^{n}$.

We will henceforth identify a vector $x=\sum x_{i} e_{i}$ with the point $x=\left(x_{i}\right)_{1}^{n}$ in $\mathbf{C}^{n}$; accordingly, the space $\mathcal{L}\left(\ell_{2}^{n}\right)$ is identified with the $C^{*}$-algebra $M_{n}(\mathbf{C})$ of complex $n \times n$ matrices.

It will be convenient to reparametrize the $K$-functional for the couple $\overline{\ell_{2}^{n}}(\lambda)$ and write

$$
k_{\lambda}(t, x):=K\left(1 / t, x ; \overline{\ell_{2}^{n}}(\lambda)\right) .
$$

By Lemma 1.1 we have

$$
k_{\lambda}(t, x)=\sum_{i=1}^{n} \frac{\lambda_{i}}{t+\lambda_{i}}\left|x_{i}\right|^{2}, \quad x \in \mathbf{C}^{n} .
$$

2.4. Basic reductions. To prove that the couple $\overline{\ell_{2}^{n}}(\lambda)$ has the $K$-property, we introduce an auxiliary parameter $\rho>1$. The exact value of $\rho$ will change meaning during the course of the argument, the main point being that it can be chosen arbitrarily close to 1 .

Initially, we pick any $\rho>1$ such that $\rho \lambda_{i}<\lambda_{i+1}$ for all $i$; we assume also that we are given two elements $x^{0}, y^{0} \in \mathbf{C}^{n}$ such that

$$
k_{\lambda}\left(t, y^{0}\right)<\frac{1}{\rho} k_{\lambda}\left(t, x^{0}\right), \quad t \geq 0 .
$$

We must construct a matrix $T \in M_{n}(\mathbf{C})$ such that

$$
T x^{0}=y^{0} \quad \text { and } \quad k_{\lambda}(t, T x) \leq k_{\lambda}(t, x), \quad x \in \mathbf{C}^{n}, t>0 .
$$


Define $\tilde{x}^{0}=\left(\left|x_{i}^{0}\right|\right)_{1}^{n}$ and $\tilde{y}^{0}=\left(\left|y_{i}^{0}\right|\right)_{1}^{n}$ and suppose that

$$
k_{\lambda}\left(t, \tilde{y}^{0}\right)<\frac{1}{\rho} k_{\lambda}\left(t, \tilde{x}^{0}\right), \quad t \geq 0
$$

Suppose that we can find an operator $T_{0} \in M_{n}(\mathbf{C})$ such that $T_{0} \tilde{x}^{0}=\tilde{y}^{0}$ and $k_{\lambda}\left(t, T_{0} x\right)<k_{\lambda}(t, x)$ for all $x \in \mathbf{C}^{n}$ and $t>0$. Writing $x_{k}^{0}=e^{i \theta_{k}} \tilde{x}_{k}^{0}$ and $y_{k}^{0}=e^{i \varphi_{k}} \tilde{y}_{k}^{0}$ where $\theta_{k}, \varphi_{k} \in \mathbf{R}$, we then have $T x^{0}=y^{0}$ and $k_{\lambda}(t, T x)<k_{\lambda}(t, x)$ where

$$
T=\operatorname{diag}\left(e^{i \varphi_{k}}\right) T_{0} \operatorname{diag}\left(e^{-i \theta_{k}}\right) .
$$

Replacing $x^{0}, y^{0}$ by $\tilde{x}^{0}, \tilde{y}^{0}$ we can thus assume that the coordinates $x_{i}^{0}$ and $y_{i}^{0}$ are non-negative; replacing them by small perturbations if necessary, we can assume that they are strictly positive, at the expense of slightly diminishing the number $\rho$.

Now put $\beta_{i}=\lambda_{i}$ and $\alpha_{i}=\rho \lambda_{i}$. Our assumption on $\rho$ means that

$$
0<\beta_{1}<\alpha_{1}<\cdots<\beta_{n}<\alpha_{n} .
$$

Using the explicit expression for the $K$-functional, it is plain to check that

$$
k_{\beta}(t, x) \leq k_{\alpha}(t, x) \leq \rho k_{\beta}(t, x), \quad x \in \mathbf{C}^{n}, t \geq 0 .
$$

Our assumption (2.6) therefore implies that

$$
k_{\alpha}\left(t, y^{0}\right)<k_{\beta}\left(t, x^{0}\right), \quad t \geq 0 .
$$

We shall verify the existence of a matrix $T=T_{\rho}=T_{\rho, x^{0}, y^{0}}$ such that

$$
T x^{0}=y^{0} \quad \text { and } \quad k_{\alpha}(t, T x) \leq k_{\beta}(t, x), \quad x \in \mathbf{C}^{n}, t>0 .
$$

It is clear by compactness that, as $\rho \downarrow 1$, the corresponding matrices $T_{\rho}$ will cluster at some point $T$ satisfying $T x^{0}=y^{0}$ and $\|T\|_{\mathcal{L}(\overline{\mathcal{H}})} \leq 1$. (See Remark 1.4)

In conclusion, the proof of Theorem 2.4 will be complete when we can construct a matrix $T$ satisfying (2.9) with $\rho$ arbitrarily close to 1 .

2.5. Construction of $T$. Let $\mathcal{P}_{k}$ denote the linear space of complex polynomials of degree at most $k$. We shall use the polynomials

$$
L_{\alpha}(t)=\prod_{1}^{n}\left(t+\alpha_{i}\right) \quad, \quad L_{\beta}(t)=\prod_{1}^{n}\left(t+\beta_{i}\right),
$$

and the product $L=L_{\alpha} L_{\beta}$. Notice that

$$
L^{\prime}\left(-\alpha_{i}\right)<0 \quad, \quad L^{\prime}\left(-\beta_{i}\right)>0 .
$$

Recalling the formula (2.5), it is clear that we can define a real polynomial $P \in$ $\mathcal{P}_{2 n-1}$ by

$$
\frac{P(t)}{L(t)}=k_{\beta}\left(t, x^{0}\right)-k_{\alpha}\left(t, y^{0}\right)
$$

Clearly $P(t)>0$ when $t \geq 0$. Moreover, a consideration of the residues at the poles of the right-hand member shows that $P$ is uniquely defined by the values

$$
P\left(-\beta_{i}\right)=\left(x_{i}^{0}\right)^{2} \beta_{i} L^{\prime}\left(-\beta_{i}\right) \quad, \quad P\left(-\alpha_{i}\right)=-\left(y_{i}^{0}\right)^{2} \alpha_{i} L^{\prime}\left(-\alpha_{i}\right) .
$$

Combining with (2.10), we conclude that

$$
P\left(-\alpha_{i}\right)>0 \text { and } P\left(-\beta_{i}\right)>0 .
$$


Perturbing the problem slightly, it is clear that we can assume that $P$ has exact degree $2 n-1$, and that all zeros of $P$ have multiplicity 1 . (We here diminish the value of $\rho>1$ somewhat, if necessary.)

Now, $P$ has $2 n-1$ simple zeros, which we split according to

$$
P^{-1}(\{0\})=\left\{-r_{i}\right\}_{i=1}^{2 m-1} \cup\left\{-c_{i},-\bar{c}_{i}\right\}_{i=1}^{n-m},
$$

where the $r_{i}$ are positive and the $c_{i}$ are non-real, and chosen to have positive imaginary parts. The following is the key observation.

Lemma 2.5. We have that

$$
L^{\prime}\left(-\beta_{i}\right) P\left(-\beta_{i}\right)>0 \quad, \quad L^{\prime}\left(-\alpha_{i}\right) P\left(-\alpha_{i}\right)<0
$$

and there is a splitting $\left\{r_{i}\right\}_{i=1}^{2 m-1}=\left\{\delta_{i}\right\}_{i=1}^{m} \cup\left\{\gamma_{i}\right\}_{i=1}^{m-1}$ such that

$$
L\left(-\delta_{j}\right) P^{\prime}\left(-\delta_{j}\right)>0 \quad, \quad L\left(-\gamma_{k}\right) P^{\prime}\left(-\gamma_{k}\right)<0 .
$$

Proof. The inequalities (2.14) follow immediately from (2.13) and (2.10). It remains to prove (2.15).

Let $-h$ denote the leftmost real zero of the polynomial $L P$ (of degree $4 n-1$ ). We claim that $P(-h)=0$. If this were not the case, we would have $h=\alpha_{n}$. Since the degree of $P$ is odd, $P(-t)$ is negative for large values of $t$, and so $P\left(-\alpha_{n}\right)<0$ contradicting (2.13). We have shown that $P(-h)=0$. Since all zeros of $L P$ have multiplicity 1 , we have $(L P)^{\prime}(-h) \neq 0$, whence

$$
L(-h) P^{\prime}(-h)=(L P)^{\prime}(-h)>0 .
$$

We write $\delta_{m}=h$ and put $P_{*}(t)=P(t) /\left(t+\delta_{m}\right)$. Since $t+\delta_{m}>0$ for $t \in$ $\left\{-\alpha_{i},-\beta_{i}\right\}_{1}^{n}$, we have by (2.13) that for all $i$

$$
P_{*}\left(-\alpha_{i}\right)>0 \text { and } P_{*}\left(-\beta_{i}\right)>0 .
$$

Denote by $\left\{-r_{j}{ }^{*}\right\}_{j=1}^{2 m-2}$ the real zeros of $P_{*}$. Since the degree of $L P_{*}$ is even and the polynomial $\left(L P_{*}\right)^{\prime}$ has alternating signs in the set $\left\{-\alpha_{i},-\beta_{i}\right\}_{i=1}^{n} \cup\left\{-r_{i}{ }^{*}\right\}_{i=1}^{2 m-2}$, we can split the zeros of $P_{*}$ as $\left\{-\delta_{i},-\gamma_{i}\right\}_{i=1}^{m-1}$, where

$$
L\left(-\delta_{i}\right) P_{*}^{\prime}\left(-\delta_{i}\right)>0 \quad, \quad L\left(-\gamma_{i}\right) P_{*}^{\prime}\left(-\gamma_{i}\right)<0 .
$$

Since $P^{\prime}\left(-r_{j}{ }^{*}\right)=\left(\delta_{m}-r_{j}{ }^{*}\right) P_{*}^{\prime}\left(-r_{j}{ }^{*}\right)$ and $\delta_{m}>r_{j}{ }^{*}$, the signs of $P^{\prime}\left(-r_{j}{ }^{*}\right)$ and $P_{*}^{\prime}\left(-r_{j}^{*}\right)$ are equal, proving (2.15).

Recall that $\left\{-c_{i}\right\}_{1}^{n-m}$ denote the zeros of $P$ such that $\operatorname{Im} c_{i}>0$. We put (with the convention that an empty product equals 1 )

$$
L_{\delta}(t)=\prod_{i=1}^{m}\left(t+\delta_{i}\right) \quad, \quad L_{\gamma}(t)=\prod_{i=1}^{m-1}\left(t+\gamma_{i}\right) \quad, \quad L_{c}(t)=\prod_{i=1}^{n-m}\left(t+c_{i}\right) .
$$

We define a linear map $F: \mathbf{C}^{n+m} \rightarrow \mathbf{C}^{n+m-1}$ in the following way. First define a subspace $U \subset \mathcal{P}_{2 n-1}$ by

$$
U=\left\{L_{c} q ; q \in \mathcal{P}_{n+m-1}\right\} .
$$

Notice that $U$ has dimension $n+m-1$ and that $P \in U$; in fact $P=a L_{c} L_{c}^{*} L_{\delta} L_{\gamma}$ where $a$ is the leading coefficient and the $*$-operation is defined by $L^{*}(z)=\overline{L(\bar{z})}$. 
For a polynomial $Q \in U$ we have

$$
\begin{aligned}
\frac{|Q(t)|^{2}}{L(t) P(t)} & =\sum_{i=1}^{n}\left|x_{i}\right|^{2} \frac{\beta_{i}}{t+\beta_{i}}+\sum_{i=1}^{n}\left|x_{i}^{\prime}\right|^{2} \frac{\delta_{i}}{t+\delta_{i}} \\
& -\sum_{i=1}^{n}\left|y_{i}\right|^{2} \frac{\alpha_{i}}{t+\alpha_{i}}-\sum_{i=1}^{m-1}\left|y_{i}^{\prime}\right|^{2} \frac{\gamma_{i}}{t+\gamma_{i}}
\end{aligned}
$$

where, for definiteness,

$$
\begin{aligned}
x_{i} & =\frac{Q\left(-\beta_{i}\right)}{\sqrt{\beta_{i} L^{\prime}\left(-\beta_{i}\right) P\left(-\beta_{i}\right)}} \quad ; \quad x_{j}^{\prime}=\frac{Q\left(-\delta_{j}\right)}{\sqrt{\delta_{j} L^{\prime}\left(-\delta_{j}\right) P\left(-\delta_{j}\right)}} \\
y_{i} & =\frac{Q\left(-\alpha_{i}\right)}{\sqrt{-\alpha_{i} L^{\prime}\left(-\alpha_{i}\right) P\left(-\alpha_{i}\right)}} \quad ; \quad y_{j}^{\prime}=\frac{Q\left(-\gamma_{j}\right)}{\sqrt{-\gamma_{j} L^{\prime}\left(-\gamma_{j}\right) P\left(-\gamma_{j}\right)}} .
\end{aligned}
$$

The identities in (2.18) give rise to a linear map

$$
M: \mathbf{C}^{n} \oplus \mathbf{C}^{m} \rightarrow U \quad ; \quad\left[x ; x^{\prime}\right] \mapsto Q .
$$

We can similarly regard (2.19) as a linear map

$$
N: U \rightarrow \mathbf{C}^{n} \oplus \mathbf{C}^{m-1} \quad ; \quad Q \mapsto\left[y ; y^{\prime}\right] .
$$

Our desired map $F$ is defined as the composite

$$
F=N M: \mathbf{C}^{n} \oplus \mathbf{C}^{m} \rightarrow \mathbf{C}^{n} \oplus \mathbf{C}^{m-1} \quad ; \quad\left[x ; x^{\prime}\right] \mapsto\left[y ; y^{\prime}\right] .
$$

Notice that if $Q=M\left[x ; x^{\prime}\right]$ and $\left[y ; y^{\prime}\right]=F\left[x ; x^{\prime}\right]$ then (2.17) means that

$$
k_{\beta \oplus \delta}\left(t,\left[x ; x^{\prime}\right]\right)-k_{\alpha \oplus \gamma}\left(t, F\left[x ; x^{\prime}\right]\right)=\frac{|Q(t)|^{2}}{L(t) P(t)} \geq 0, \quad t \geq 0 .
$$

This implies that $F$ is a contraction from $\overline{\ell_{2}^{n+m}}(\beta \oplus \delta)$ to $\overline{\ell_{2}^{n+m-1}}(\alpha \oplus \gamma)$.

We now define $T$ as a "compression" of $F$. Namely, let $E: \mathbf{C}^{n} \oplus \mathbf{C}^{m-1} \rightarrow \mathbf{C}^{n}$ be the projection onto the first $n$ coordinates, and define an operator $T$ on $\mathbf{C}^{n}$ by

$$
T x=E F[x ; 0], \quad x \in \mathbf{C}^{n} .
$$

Taking $Q=P$ in (2.17) we see that $T x^{0}=y^{0}$. Moreover,

$$
\begin{aligned}
k_{\beta}(t, x)-k_{\alpha}(t, T x) & =\sum_{i=1}^{n}\left|x_{i}\right|^{2} \frac{\beta_{i}}{t+\beta_{i}}-\sum_{i=1}^{n}\left|y_{i}\right|^{2} \frac{\alpha_{i}}{t+\alpha_{i}} \\
& \geq \sum_{i=1}^{n}\left|x_{i}\right|^{2} \frac{\beta_{i}}{t+\beta_{i}}-\sum_{i=1}^{n}\left|y_{i}\right|^{2} \frac{\alpha_{i}}{t+\alpha_{i}}-\sum_{j=1}^{m-1}\left|y_{i}^{\prime}\right|^{2} \frac{\gamma_{i}}{t+\gamma_{i}} \\
& =k_{\beta \oplus \delta}(t,[x ; 0])-k_{\alpha \oplus \gamma}(t, F[x ; 0])=\frac{|Q(t)|^{2}}{L(t) P(t)} .
\end{aligned}
$$

Since the right-hand side is non-negative, we have shown that

$$
k_{\alpha}(t, T x) \leq k_{\beta}(t, x), \quad t>0, x \in \mathbf{C}^{n},
$$

as desired. The proof of Theorem 2.4 is finished. q.e.d. 
2.6. Real scalars. Theorem 2.4 holds also in the case of Euclidean spaces over the real scalar field. To see this, assume without loss of generality that the vectors $x^{0}, y^{0} \in \mathbf{C}^{n}$ have real entries (still satisfying $k_{\lambda}\left(t, y^{0}\right) \leq k_{\lambda}\left(t, x^{0}\right)$ for all $t>0$ ).

By Theorem 2.4 we can find a (complex) contraction $T$ of $\overline{\ell_{2}^{n}}(\lambda)$ such that $T x^{0}=$ $y^{0}$. It is clear that the operator $T^{*}$ defined by $T^{*} x=\overline{T(\bar{x})}$ satisfies those same conditions. Replacing $T$ by $\frac{1}{2}\left(T+T^{*}\right)$ we obtain a real matrix $T \in M_{n}(\mathbf{R})$, which is a contraction of $\overline{\ell_{2}^{n}}(\lambda)$ and maps $x^{0}$ to $y^{0}$.

2.7. Explicit representations. We here deduce an explicit representation for the operator $T$ constructed above.

Let $x^{0}$ and $y^{0}$ be two non-negative vectors such that

$$
k_{\lambda}\left(t, y^{0}\right) \leq k_{\lambda}\left(t, x^{0}\right), \quad t>0 .
$$

For small $\rho>0$ we perturb $x^{0}, y^{0}$ slightly to vectors $\tilde{x}^{0}, \tilde{y}^{0}$ which satisfy the conditions imposed the previous subsections. We can then construct a matrix $T=T_{\rho}$ such that

$$
T \tilde{x}^{0}=\tilde{y}^{0} \quad \text { and } \quad k_{\alpha}(t, T x) \leq k_{\beta}(t, x), \quad t>0, x \in \mathbf{C}^{n},
$$

where $\beta=\lambda$ and $\alpha=\rho \lambda$. As $\rho, \tilde{x}^{0}, \tilde{y}^{0}$ approaches $1, x^{0}$, resp. $y^{0}$, it is clear that any cluster point $T$ of the set of contractions $T_{\rho}$ will satisfy

$$
T x^{0}=y^{0} \quad \text { and } \quad k_{\lambda}(t, T x) \leq k_{\lambda}(t, x), \quad t>0, x \in \mathbf{C}^{n} .
$$

Theorem 2.6. The matrix $T=T_{\varrho}=\left(\tau_{i k}\right)_{i, k=1}^{n}$ where

$$
\tau_{i k}=\operatorname{Re}\left[\frac{1}{\alpha_{i}-\beta_{k}} \frac{\tilde{x}_{k}^{0}}{\tilde{y}_{i}^{0}} \frac{\beta_{k} L_{\delta}\left(-\alpha_{i}\right) L_{c}\left(-\alpha_{i}\right) L_{\alpha}\left(-\beta_{k}\right)}{\alpha_{i} L_{\delta}\left(-\beta_{k}\right) L_{c}\left(-\beta_{k}\right) L_{\alpha}^{\prime}\left(-\alpha_{i}\right)}\right]
$$

satisfies (2.22).

Proof. The range of the map $\mathbf{C}^{n} \rightarrow U, x \mapsto M[x ; 0]$ (see 2.20) is precisely the $n$-dimensional subspace

$$
V:=L_{\delta} L_{c} \cdot \mathcal{P}_{n-1}=\left\{L_{\delta} L_{c} R ; R \in \mathcal{P}_{n-1}\right\} \subset U .
$$

We introduce a basis $\left(Q_{k}\right)_{k=1}^{n}$ for $V$ by

$$
Q_{k}(t)=\frac{L_{\delta}(t) L_{c}(t) L_{\beta}(t)}{t+\beta_{k}} \frac{\sqrt{\beta_{k} L^{\prime}\left(-\beta_{k}\right) P\left(-\beta_{k}\right)}}{L_{\delta}\left(-\beta_{k}\right) L_{c}\left(-\beta_{k}\right) L_{\beta}^{\prime}\left(-\beta_{k}\right)} .
$$

Then

$$
\frac{Q_{k}\left(-\beta_{i}\right)}{\sqrt{\beta_{i} L^{\prime}\left(-\beta_{i}\right) P\left(-\beta_{i}\right)}}= \begin{cases}1 & i=k \\ 0 & i \neq k\end{cases}
$$

Denoting by $\left(e_{i}\right)$ the canonical basis in $\mathbf{C}^{n}$ and using (2.18), (2.19) we get

$$
\begin{aligned}
\tau_{i k} & =\left(T e_{k}\right)_{i}=\frac{Q_{k}\left(-\alpha_{i}\right)}{\sqrt{\alpha_{i} L^{\prime}\left(-\alpha_{i}\right) P\left(-\alpha_{i}\right)}} \\
& =\frac{1}{\beta_{k}-\alpha_{i}} \frac{L_{\delta}\left(-\alpha_{i}\right) L_{c}\left(-\alpha_{i}\right) L_{\beta}\left(-\alpha_{i}\right)}{L_{\delta}\left(-\beta_{k}\right) L_{c}\left(-\beta_{k}\right) L_{\beta}^{\prime}\left(-\beta_{k}\right)}\left(\frac{\beta_{k} L^{\prime}\left(-\beta_{k}\right) P\left(-\beta_{k}\right)}{-\alpha_{i} L^{\prime}\left(-\alpha_{i}\right) P\left(-\alpha_{i}\right)}\right)^{1 / 2} .
\end{aligned}
$$

Inserting the expressions (2.12) for $P\left(-\alpha_{i}\right)$ and $P\left(-\beta_{k}\right)$ and taking real parts (see the remarks in $\$ 2.6)$, we obtain the formula (2.23). 
Remark 2.7. It is easy to see that, if we pick all matrix-elements real, some elements $\tau_{i k}$ of the matrix $T$ in (2.23) will be negative, even while the numbers $x_{i}^{0}$ and $y_{k}^{0}$ are positive. It was proved in [2, Theorem 2.3, that this is necessarily so. Indeed, one there constructs an example of a five-dimensional couple $\overline{\ell_{2}^{5}}(\lambda)$ and two vectors $x^{0}, y^{0} \in \mathbf{R}^{5}$ having non-negative entries such that no contraction $T=\left(\tau_{i k}\right)_{i, k=1}^{5}$ on $\overline{\ell_{2}^{5}}(\lambda)$ having all matrix entries $\tau_{i k} \geq 0$ can satisfy $T x^{0}=y^{0}$. On the other hand, if one settles for using a matrix with $\|T\| \leq \sqrt{2}$, then it is possible to find one with only non-negative matrix entries. Indeed, such a matrix was used by Sedaev [35, see also [39].

2.8. On sharpness of the norm-bounds. We shall show that if $m<n$ (i.e. if the polynomial $P$ has at least one non-real zero), then the norm $\|T\|_{\mathcal{L}\left(\mathcal{H}_{i}\right)}$ of the contraction $T$ constructed above is very close to 1 for $i=0,1$.

We first claim that $\|T\|_{\mathcal{L}\left(\mathcal{H}_{0}\right)}=1$. To see this, we notice that if $m<n$, then there is a non-trivial polynomial $Q^{(1)}$ in the space $V$ (see (2.24) $)$ which vanishes at the points $0, \gamma_{1}, \ldots, \gamma_{m-1}$. If $x_{i}^{(1)}$ and $y_{i}^{(1)}$ are defined by the formulas (2.18) and (2.19) (while $\left.\left(x_{j}^{(1)}\right)^{\prime}=\left(y_{k}^{(1)}\right)^{\prime}=0\right)$, we then have $T x^{(1)}=y^{(1)}$ and

$$
k_{\beta}\left(t, x^{(1)}\right)-k_{\alpha}\left(t, y^{(1)}\right)=\frac{\left|Q^{(1)}(t)\right|^{2}}{L(t) P(t)}, \quad t>0 .
$$

Choosing $t=0$ we conclude that $\left\|x^{(1)}\right\|_{\ell_{2}^{n}}^{2}-\left\|T x^{(1)}\right\|_{\ell_{2}^{n}}^{2}=0$, whence $\|T\|_{\mathcal{L}\left(\mathcal{H}_{0}\right)} \geq 1$, proving our claim.

Similarly, the condition $m<n$ implies the existence of a polynomial $Q^{(2)} \in V$ of degree at most $n+m-2$ vanishing at the points $\gamma_{1}, \ldots, \gamma_{m-1}$. Constructing vectors $x^{(2)}, y^{(2)}$ via (2.18) and (2.19) we will have $T x^{(2)}=y^{(2)}$ and

$$
k_{\beta}\left(t, x^{(2)}\right)-k_{\alpha}\left(t, y^{(2)}\right)=\frac{\left|Q^{(2)}(t)\right|^{2}}{L(t) P(t)}, \quad t>0 .
$$

Multiplying this relation by $t$ and then sending $t \rightarrow \infty$, we find that $\left\|x^{(2)}\right\|_{\ell_{2}^{n}(\beta)}^{2}-$ $\left\|T x^{(2)}\right\|_{\ell_{2}^{n}(\alpha)}^{2}=0$, which implies $\|T\|_{\mathcal{L}\left(\mathcal{H}_{1}\right)} \geq \rho^{-1 / 2}$.

2.9. A remark on weighted $\ell_{p}$-couples. As far as we are aware, if $1<p<\infty$ and $p \neq 2$, it is still an open question whether the couple $\overline{\ell_{p}^{n}}(\lambda)=\left(\ell_{p}^{n}, \ell_{p}^{n}(\lambda)\right)$ is an exact Calderón couple or not. (When $p=1$ or $p=\infty$ it is exact Calderón; see [36] for the case $p=1$; the case $p=\infty$ is essentially just the Hahn-Banach theorem.)

It is well known, and easy to prove, that the $K_{p}$-functional (see (2.2) ) corresponding to the couple $\overline{\ell_{p}^{n}}(\lambda)$ is given by the explicit formula

$$
K_{p}\left(t, x ; \overline{\ell_{p}^{n}}(\lambda)\right)=\sum_{i=1}^{n}\left|x_{i}\right|^{p} \frac{t \lambda_{i}}{\left(1+\left(t \lambda_{i}\right)^{\frac{1}{p-1}}\right)^{p-1}} .
$$

It was proved by Sedaev [35] (cf. [39]) that if $K_{p}\left(t, y^{0} ; \overline{\ell_{p}^{n}}(\lambda)\right) \leq K_{p}\left(t, x^{0} ; \overline{\ell_{p}^{n}}(\lambda)\right)$ for all $t>0$ then there is $T: \overline{\ell_{p}^{n}}(\lambda) \rightarrow \overline{\ell_{p}^{n}}(\lambda)$ of norm at most $2^{1 / p^{\prime}}$ such that $T x^{0}=y^{0}$. (Here $p^{\prime}$ is the exponent conjugate to $p$.) 
Although our present estimates are particular for the case $p=2$, our construction still shows that, if we re-define $P(t)$ to be the polynomial

$$
\frac{P(t)}{L(t)}=\sum_{1}^{n}\left(\tilde{x}_{i}^{0}\right)^{p} \frac{\beta_{i}}{t+\beta_{i}}-\sum_{1}^{n}\left(\tilde{y}_{i}^{0}\right)^{p} \frac{\alpha_{i}}{t+\alpha_{i}}
$$

then the matrix $T$ defined by

$$
\tau_{i k}=\operatorname{Re}\left[\frac{1}{\alpha_{i}-\beta_{k}} \frac{\left(\tilde{x}_{k}^{0}\right)^{p-1}}{\left(\tilde{y}_{i}^{0}\right)^{p-1}} \frac{\beta_{k} L_{\delta}\left(-\alpha_{i}\right) L_{c}\left(-\alpha_{i}\right) L_{\alpha}\left(-\beta_{k}\right)}{\alpha_{i} L_{\delta}\left(-\beta_{k}\right) L_{c}\left(-\beta_{k}\right) L_{\alpha}^{\prime}\left(-\alpha_{i}\right)}\right]
$$

will satisfy $T \tilde{x}^{0}=\tilde{y}^{0}$, at least, provided that $P(t)>0$ when $t \geq 0$. (Here $L_{\delta}$ and $L_{c}$ are constructed from the zeros of $P$ as in the case $p=2$.)

The matrix (2.26) differs from those used by Sedaev 35] and Sparr 39. Indeed the matrices from [35, 39] have non-negative entries, while this is not so for the matrices (2.26). It seems to be an interesting problem to estimate the norm $\|T\|_{\mathcal{L}\left(\overline{\ell_{p}^{n}}(\lambda)\right)}$ for the matrix (2.26), when $p \neq 2$. The motivation for this type of question is somewhat elaborated in $\$ 6.7$, but we shall not discuss it further here.

2.10. A comparison with Löwner's matrix. In this subsection, we briefly explain how our matrix $T$ is related to the matrix used by Löwner [26] in his original work on monotone matrix functions. (2)

We shall presently display four kinds of partial isometries; Löwner's matrix will be recognized as one of them. In all cases, operators with the required properties can alternatively be found using the more general construction in Theorem 2.4.

The following discussion was inspired by the earlier work of Sparr 38, who seems to have been the first to note that Löwner's matrix could be constructed in a similar way.

In this subsection, scalars are assumed to be real. In particular, when we write " $\ell_{2}^{n "}$ we mean the (real) Euclidean $n$-dimensional space.

Suppose that two vectors $x^{0}, y^{0} \in \mathbf{R}^{n}$ satisfy

$$
k_{\lambda}\left(t, y^{0}\right) \leq k_{\lambda}\left(t, x^{0}\right), \quad t>0 .
$$

Let

$$
L_{\lambda}(t)=\prod_{1}^{n}\left(t+\lambda_{i}\right),
$$

and let $P \in \mathcal{P}_{n-1}$ be the polynomial fulfilling

$$
\frac{P(t)}{L_{\lambda}(t)}=k_{\lambda}\left(t, x^{0}\right)-k_{\lambda}\left(t, y^{0}\right)=\sum_{i=1}^{n} \frac{\lambda_{i}}{t+\lambda_{i}}\left[\left(x_{i}^{0}\right)^{2}-\left(y_{i}^{0}\right)^{2}\right] \text {. }
$$

By assumption, $P(t) \geq 0$ for $t \geq 0$. Moreover, $P$ is uniquely determined by the $n$ conditions

$$
P\left(-\lambda_{i}\right)=\frac{\left(x_{i}^{0}\right)^{2}-\left(y_{i}^{0}\right)^{2}}{\lambda_{i} L_{\lambda}^{\prime}\left(-\lambda_{i}\right)} .
$$

Let $u_{1}, v_{1}, u_{2}, v_{2}, \ldots$ denote the canonical basis of $\ell_{2}^{n}$ and let

$$
\ell_{2}^{n}=O \oplus E
$$

\footnotetext{
${ }^{2}$ By "Löwner's matrix", we mean the unitary matrix denoted " $V$ " in Donoghue's book [12], on p. 71. A more explicit construction of this matrix is found in [26], where it is called "T".
} 
be the corresponding splitting, i.e.,

$$
O=\operatorname{span}\left\{u_{i}\right\} \quad, \quad E=\operatorname{span}\left\{v_{i}\right\} .
$$

Notice that

$$
\operatorname{dim} O=\lfloor(n-1) / 2\rfloor+1 \quad, \quad \operatorname{dim} E=\lfloor(n-2) / 2\rfloor+1,
$$

where $\lfloor x\rfloor$ is the integer part of a real number $x$.

We shall construct matrices $T \in M_{n}(\mathbf{R})$ such that

$$
T x^{0}=y^{0} \quad \text { and } \quad k_{\lambda}(t, T x) \leq k_{\lambda}(t, x), \quad t>0, x \in \mathbf{R}^{n},
$$

in the following special cases:

(1) $P(t)=q(t)^{2}$ where $q \in \mathcal{P}_{(n-1) / 2}(\mathbf{R}), x^{0} \in O$, and $y^{0} \in E$,

(2) $P(t)=t q(t)^{2}$ where $q \in \mathcal{P}_{(n-2) / 2}(\mathbf{R}), x^{0} \in E$, and $y^{0} \in O$.

Here $\mathcal{P}_{x}$ should be interpreted as $\mathcal{P}_{\lfloor x\rfloor}$.

Remark 2.8. In this connection, it is interesting to recall the well-known fact that any polynomial $P$ which is non-negative on $\mathbf{R}_{+}$can be written $P(t)=q_{0}(t)^{2}+$ $t q_{1}(t)^{2}$ for some real polynomials $q_{0}$ and $q_{1}$.

To proceed with the solution, we rename the $\lambda_{i}$ as $\lambda_{i}=\xi_{i}$ when $i$ is odd and $\lambda_{i}=\eta_{i}$ when $i$ is even. We also write

$$
L_{\xi}(t)=\prod_{i \text { odd }}\left(t+\xi_{i}\right) \quad, \quad L_{\eta}(t)=\prod_{i \text { even }}\left(t+\eta_{i}\right),
$$

and write $L=L_{\xi} L_{\eta}$. Notice that $L_{\lambda}^{\prime}\left(-\xi_{i}\right)>0$ and $L_{\lambda}^{\prime}\left(-\eta_{i}\right)<0$.

Case 1. Suppose that $P(t)=q(t)^{2}, q \in \mathcal{P}_{(n-1) / 2}(\mathbf{R}), x^{0} \in O$, and $y^{0} \in E$. Then

$$
\frac{q(t)^{2}}{L_{\lambda}(t)}=\sum_{k \text { odd }} \frac{\xi_{k}}{t+\xi_{k}}\left(x_{k}^{0}\right)^{2}-\sum_{i \text { even }} \frac{\eta_{i}}{t+\eta_{i}}\left(y_{i}^{0}\right)^{2},
$$

where

$$
x_{k}^{0}=\frac{\varepsilon_{k} q\left(-\xi_{k}\right)}{\sqrt{\xi_{k} L_{\lambda}^{\prime}\left(-\xi_{k}\right)}} \quad, \quad y_{i}^{0}=\frac{\zeta_{i} q\left(-\eta_{i}\right)}{\sqrt{-\eta_{i} L_{\lambda}^{\prime}\left(-\eta_{i}\right)}}
$$

for some choice of signs $\varepsilon_{k}, \zeta_{i} \in\{ \pm 1\}$.

By (2.28) are defined linear maps

$$
O \rightarrow \mathcal{P}_{(n-1) / 2}(\mathbf{R}) \quad: \quad x \mapsto Q \quad ; \quad \mathcal{P}_{(n-1) / 2}(\mathbf{R}) \rightarrow E \quad: \quad Q \mapsto y .
$$

The composition is a linear map

$$
T_{0}: O \rightarrow E \quad: \quad x \mapsto y .
$$

We now define $T \in M_{n}(\mathbf{R})$ by

$$
T: O \oplus E \rightarrow O \oplus E \quad: \quad[x ; v] \mapsto\left[0 ; T_{0} x\right] .
$$

Then clearly $T x^{0}=y^{0}$ and

$$
\begin{aligned}
k_{\lambda}(t,[x ; v]) & -k_{\lambda}(t, T[x ; v]) \\
& \geq k_{\xi}(t, x)-k_{\eta}\left(t, T_{0} x\right) \\
& =\frac{Q(t)^{2}}{L_{\lambda}(t)} \geq 0, \quad t>0, x \in O, v \in E .
\end{aligned}
$$


We have verified (2.27) in case 1. A computation similar to the one in the proof of Theorem 2.6 shows that, with respect to the bases $u_{k}$ and $v_{i}$,

$$
\left(T_{0}\right)_{i k}=\frac{\varepsilon_{k} \zeta_{i}}{\xi_{k}-\eta_{i}} \frac{L_{\xi}\left(-\eta_{i}\right)}{L_{\xi}^{\prime}\left(-\xi_{k}\right)}\left(\frac{\xi_{k} L_{\xi}^{\prime}\left(-\xi_{k}\right) L_{\eta}\left(-\xi_{k}\right)}{-\eta_{i} L_{\xi}\left(-\eta_{i}\right) L_{\eta}^{\prime}\left(-\eta_{i}\right)}\right)^{1 / 2} .
$$

Notice that, multiplying (2.29) by $t$, then letting $t \rightarrow \infty$ implies that

$$
\sum_{k \text { odd }} x_{k}^{2} \xi_{k}-\sum_{i \text { even }}\left(T_{0} x\right)_{i}^{2} \eta_{i}=0
$$

This means that $T$ is a partial isometry from $O$ to $E$ with respect to the norm of $\ell_{2}^{n}(\lambda)$

Case 2. Now assume that $P(t)=t q(t)^{2}, q \in \mathcal{P}_{(n-2) / 2}(\mathbf{R}), x^{0} \in E$, and $y^{0} \in O$. Then

where

$$
\frac{t q(t)^{2}}{L_{\lambda}(t)}=-\sum_{i \text { odd }}\left(y_{i}^{0}\right)^{2} \frac{\xi_{i}}{t+\xi_{i}}+\sum_{k \text { even }} \frac{\eta_{k}}{t+\eta_{k}}\left(x_{k}^{0}\right)^{2}
$$

for some $\varepsilon_{i}^{\prime}, \zeta_{k}^{\prime} \in\{ \pm 1\}$.

By (2.30) are defined linear maps

$$
E \rightarrow \mathcal{P}_{(n-2) / 2}(\mathbf{R}) \quad: \quad x \mapsto Q \quad ; \quad \mathcal{P}_{(n-2) / 2}(\mathbf{R}) \rightarrow O \quad: \quad Q \mapsto y .
$$

We denote their composite by

$$
T_{1}: E \rightarrow O \quad: \quad x \mapsto y .
$$

Define $T \in M_{n}(\mathbf{R})$ by

$$
T: O \oplus E \rightarrow O \oplus E \quad: \quad[u ; x] \mapsto\left[T_{1} x ; 0\right] .
$$

We then have

$$
\begin{aligned}
-k_{\lambda}(t, T[u ; x]) & +k_{\lambda}(t,[u ; x]) \\
& \geq-k_{\xi}\left(t, T_{1} x\right)+k_{\eta}(t, x) \\
& =\frac{t Q(t)^{2}}{L_{\lambda}(t)} \geq 0, \quad t>0, u \in O, x \in E
\end{aligned}
$$

and (2.27) is verified also in case 2 ,

A computation shows that, with respect to the bases $v_{k}$ and $u_{i}$,

$$
\left(T_{1}\right)_{i k}=\frac{\varepsilon_{i}^{\prime} \zeta_{k}^{\prime}}{\eta_{k}-\xi_{i}} \frac{L_{\eta}\left(-\xi_{i}\right)}{L_{\eta}^{\prime}\left(-\eta_{k}\right)}\left(\frac{-L_{\xi}\left(-\eta_{k}\right) L_{\eta}^{\prime}\left(-\eta_{k}\right)}{L_{\xi}^{\prime}\left(-\xi_{i}\right) L_{\eta}\left(-\xi_{i}\right)}\right)^{1 / 2}
$$

Inserting $t=0$ in (2.31) we find that

$$
-\sum_{i \text { odd }}\left(T_{1} x\right)_{i}^{2}+\sum_{k \text { even }}\left(x_{k}\right)^{2}=0
$$

i.e., $T$ is a partial isometry form $E$ to $O$ with respect to the norm of $\ell_{2}^{n}$.

In the case of even $n$, the matrix $T_{1}$ coincides with Löwner's matrix. 


\section{QuAdratic InTERPolation SPACES}

3.1. A classification of quadratic interpolation spaces. Recall that an intermediate space $X$ with respect to $\overline{\mathcal{H}}$ is said to be of type $H$ if $\|T\|_{\mathcal{L}\left(\mathcal{H}_{i}\right)} \leq M_{i}$ for $i=0,1$ implies that $\|T\|_{\mathcal{L}(X)} \leq H\left(M_{0}, M_{1}\right)$. We shall henceforth make a mild restriction, and assume that $H$ be homogeneous of degree one. This means that we can write

$$
H(s, t)^{2}=s^{2} \mathbf{H}\left(t^{2} / s^{2}\right)
$$

for some function $\mathbf{H}$ of one positive variable. In this situation, we will say that $X$ is of type $\mathbf{H}$. The definition is chosen so that the estimates $\|T\|_{\mathcal{L}\left(\mathcal{H}_{i}\right)}^{2} \leq M_{i}$ for $i=0,1$ imply $\|T\|_{\mathcal{L}(X)}^{2} \leq M_{0} \mathbf{H}\left(M_{1} / M_{0}\right)$.

In the following we will make the standing assumptions: $\mathbf{H}$ is an increasing, continuous, and positive function on $\mathbf{R}_{+}$with $\mathbf{H}(1)=1$ and $\mathbf{H}(t) \leq \max \{1, t\}$.

Notice that our assumptions imply that all spaces of type $\mathbf{H}$ are exact interpolation. Note also that $\mathbf{H}(t)=t^{\theta}$ corresponds to geometric interpolation of exponent $\theta$.

Suppose now that $\overline{\mathcal{H}}$ is a regular Hilbert couple and that $\mathcal{H}_{*}$ is an exact interpolation space with corresponding operator $B$. By Donoghue's lemma, we have that $B=h(A)$ for some positive Borel function $h$ on $\sigma(A)$.

The statement that $\mathcal{H}_{*}$ is intermediate relative to $\overline{\mathcal{H}}$ is equivalent to that

$$
c_{1} \frac{A}{1+A} \leq B \leq c_{2}(1+A)
$$

for some positive numbers $c_{1}$ and $c_{2}$.

Let us momentarily assume that $\mathcal{H}_{0}$ be separable. (This restriction is removed in Remark 3.1]) We can then define the scalar-valued spectral measure of $A$,

$$
\nu_{A}(\omega)=\sum 2^{-k}\left\langle E(\omega) e_{k}, e_{k}\right\rangle_{0}
$$

where $E$ is the spectral measure of $A,\left\{e_{k} ; k=1,2, \ldots\right\}$ is an orthonormal basis for $\mathcal{H}_{0}$, and $\omega$ is a Borel set. Then, for Borel functions $h_{0}, h_{1}$ on $\sigma(A)$, one has that $h_{1}=h_{2}$ almost everywhere with respect to $\nu_{A}$ if and only if $h_{1}(A)=h_{2}(A)$.

Note that the regularity of $\overline{\mathcal{H}}$ means that $\nu_{A}(\{0\})=0$.

Theorem II. If $\mathcal{H}_{*}$ is of type $\mathbf{H}$ with respect to $\overline{\mathcal{H}}$, then $B=h(A)$ where the function $h$ can be modified on a null-set with respect to $\nu_{A}$ so that

$$
h(\lambda) / h(\mu) \leq \mathbf{H}(\lambda / \mu), \quad \lambda, \mu \in \sigma(A) \backslash\{0\} .
$$

Proof. Fix a (large) compact subset $K \subset \sigma(A) \cap \mathbf{R}_{+}$and put $\mathcal{H}_{0}^{\prime}=\mathcal{H}_{1}^{\prime}=E_{K}\left(\mathcal{H}_{0}\right)$ where $E$ is the spectral measure of $A$, and the norms are defined by restriction,

$$
\|x\|_{\mathcal{H}_{i}^{\prime}}=\|x\|_{\mathcal{H}_{i}} \quad, \quad\|x\|_{\mathcal{H}_{*}^{\prime}}=\|x\|_{\mathcal{H}_{*}} \quad, \quad x \in E_{K}\left(\mathcal{H}_{0}\right) .
$$

It is clear that the operator $A^{\prime}$ corresponding to $\overline{\mathcal{H}^{\prime}}$ is the compression of $A$ to $\mathcal{H}_{0}^{\prime}$ and likewise the operator $B^{\prime}$ corresponding to $\mathcal{H}_{*}^{\prime}$ is the compression of $B$ to $\mathcal{H}_{0}^{\prime}$. Moreover, $\mathcal{H}_{*}^{\prime}$ is of interpolation type $\mathbf{H}$ with respect to $\overline{\mathcal{H}^{\prime}}$ and the operator $B^{\prime}=\left(\left.h\right|_{K}\right)\left(A^{\prime}\right)$. For this reason, and since the compact set $K$ is arbitrary, it clearly suffices to prove the statement with $\overline{\mathcal{H}}$ replaced by $\overline{\mathcal{H}^{\prime}}$. Then $A$ is bounded above and below. Moreover, by (3.2), also $B$ is bounded above and below. 
Let $c<1$ be a positive number such that $\sigma(A) \subset\left(c, c^{-1}\right)$. For a fixed $\varepsilon>0$ with $\varepsilon<c / 2$ we set

$$
E_{\lambda}=\sigma(A) \cap(\lambda-\varepsilon, \lambda+\varepsilon)
$$

and consider the functions

$$
m_{\varepsilon}(\lambda)=\operatorname{essinf}_{E_{\lambda}} h, \quad M_{\varepsilon}(\lambda)=\operatorname{ess}_{\sup _{E_{\lambda}}} h,
$$

the essential inf and sup being taken with respect to $\nu_{A}$.

Now fix a small positive number $\varepsilon^{\prime}$ and two unit vectors $e_{\lambda}, e_{\mu}$ supported by $E_{\lambda}, E_{\mu}$ respectively, such that

$$
\left\|e_{\lambda}\right\|_{*}^{2} \geq M_{\varepsilon}(\lambda)-\varepsilon^{\prime}, \quad\left\|e_{\mu}\right\|_{*}^{2} \leq m_{\varepsilon}(\mu)+\varepsilon^{\prime} .
$$

Now fix $\lambda, \mu \in \sigma(A)$ and let $T x=\left\langle x, e_{\mu}\right\rangle_{0} e_{\lambda}$. Then

$$
\begin{aligned}
\|T x\|_{1}^{2} & =\left|\left\langle x, e_{\mu}\right\rangle_{0}\right|^{2}\left\|e_{\lambda}\right\|_{1}^{2} \leq \frac{1}{(\mu-\varepsilon)^{2}}\left|\left\langle x, e_{\mu}\right\rangle_{1}\right|^{2}(\lambda+\varepsilon) \\
& \leq \frac{(\mu+\varepsilon)(\lambda+\varepsilon)}{(\mu-\varepsilon)^{2}}\|x\|_{1}^{2} .
\end{aligned}
$$

Likewise,

$$
\|T x\|_{0}^{2} \leq\left|\left\langle x, e_{\mu}\right\rangle_{0}\right|^{2} \leq\|x\|_{0}^{2}
$$

so $\|T\| \leq 1$ and $\|T\|_{A}^{2} \leq \alpha_{\mu, \lambda, \varepsilon}$ where $\alpha_{\mu, \lambda, \varepsilon}=\frac{(\mu+\varepsilon)(\lambda+\varepsilon)}{(\mu-\varepsilon)^{2}}$.

Since $\mathcal{H}_{*}$ is of type $\mathbf{H}$, we conclude that

$$
\|T\|_{B}^{2} \leq \mathbf{H}\left(\alpha_{\mu, \lambda, \varepsilon}\right),
$$

whence

$$
\begin{aligned}
M_{\varepsilon}(\lambda)-\varepsilon^{\prime} & \leq\left\|e_{\lambda}\right\|_{*}^{2}=\left\|T e_{\mu}\right\|_{*}^{2} \leq \mathbf{H}\left(\alpha_{\mu, \lambda, \varepsilon}\right)\left\|e_{\mu}\right\|_{*}^{2} \\
& \leq \mathbf{H}\left(\alpha_{\mu, \lambda, \varepsilon}\right)\left(m_{\varepsilon}(\mu)+\varepsilon^{\prime}\right) .
\end{aligned}
$$

In particular, since $\varepsilon^{\prime}$ was arbitrary, and $m_{\varepsilon}(\lambda) \leq\left\|e_{\lambda}\right\|_{*}^{2} \leq\|B\|$, we find that

$$
M_{\varepsilon}(\lambda)-m_{\varepsilon}(\lambda) \leq\left[\mathbf{H}\left(\alpha_{\mu, \lambda, \varepsilon}\right)-1\right]\|B\| .
$$

By assumption, $\mathbf{H}$ is continuous and $\mathbf{H}(1)=1$. Hence, as $\varepsilon \downarrow 0$, the functions $M_{\varepsilon}(\lambda)$ diminish monotonically, converging uniformly to a function $h_{*}(\lambda)$ which is also the uniform limit of the family $m_{\varepsilon}(\lambda)$. It is clear that $h_{*}$ is continuous, and since $m_{\varepsilon} \leq h_{*} \leq M_{\varepsilon}$, we have $h_{*}=h$ almost everywhere with respect to $\nu_{A}$. The relation (3.3) now follows for $h=h_{*}$ by letting $\varepsilon$ and $\varepsilon^{\prime}$ tend to zero in (3.4).

A partial converse to Theorem $\amalg$ is found below, see Theorem 6.3 .

Remark 3.1. (The non-separable case.) Now consider the case when $\mathcal{H}_{0}$ is nonseparable. (By regularity this means that also $\mathcal{H}_{1}$ and $\mathcal{H}_{*}$ are non-separable.)

First assume that the operator $A$ is bounded. Let $\mathcal{H}_{0}^{\prime}$ be a separable reducing subspace for $A$ such that the restriction $A^{\prime}$ of $A$ to $\mathcal{H}_{0}^{\prime}$ has the same spectrum as $A$. The space $\mathcal{H}_{0}^{\prime}$ reduces $B$ by Donoghue's lemma; by Theorem $\Pi$ the restriction $B^{\prime}$ of $B$ to $\mathcal{H}_{0}^{\prime}$ satisfies $B^{\prime}=h^{\prime}\left(A^{\prime}\right)$ for some continuous function $h^{\prime}$ satisfying (3.3) on $\sigma(A)$. Let $\mathcal{H}_{0}^{\prime \prime}$ be any other separable reducing subspace, where (as before) $B^{\prime \prime}=h^{\prime \prime}\left(A^{\prime \prime}\right)$. Then $\mathcal{H}_{0}^{\prime} \oplus \mathcal{H}_{0}^{\prime \prime}$ is a separable reducing subspace on which $B=h(A)$ for some third continuous function $h$ on $\sigma(A)$. Then $h\left(A^{\prime}\right) \oplus h\left(A^{\prime \prime}\right)=h^{\prime}\left(A^{\prime}\right) \oplus h^{\prime \prime}\left(A^{\prime \prime}\right)$ and by continuity we must have $h=h^{\prime}=h^{\prime \prime}$ on $\sigma(A)$. The function $h$ thus satisfies $B=h(A)$ as well as the estimate (3.3). 
If $A$ is unbounded, we replace $A$ by its compression to $P_{n} \mathcal{H}_{0}$ where $P_{n}$ is the spectral projection of $A$ corresponding to the spectral set $[0, n] \cap \sigma(A), n=1,2, \ldots$. The same reasoning as above shows that $B$ appears as a continuous function of $A$ on $\sigma(A) \cap[0, n]$. Since $n$ is arbitrary, we find that $B=h(A)$ for a function $h$ satisfying (3.3).

3.2. Geometric interpolation. Now consider the particular case when $\mathcal{H}_{*}$ is of exponent $\theta$, viz. of type $\mathbf{H}(t)=t^{\theta}$ with respect to $\overline{\mathcal{H}}$. We write $B=h(A)$ where $h$ is the continuous function provided by Theorem [II (and Remark 3.1 in the nonseparable case).

Fix a point $\lambda_{0} \in \sigma(A)$ and let $C=h\left(\lambda_{0}\right) \lambda_{0}^{-\theta}$. The estimate (3.3) then implies that $h(\lambda) \leq C \lambda^{\theta}$ and $h(\mu) \geq C \mu^{\theta}$ for all $\lambda, \mu \in \sigma(A)$. We have proved the following theorem.

Theorem 3.2. (27, 40]) If $\mathcal{H}_{*}$ is an exact interpolation Hilbert space of exponent $\theta$ relative to $\overline{\mathcal{H}}$, then $B=h(A)$ where $h(\lambda)=C \lambda^{\theta}$ for some positive constant $C$.

Theorem 3.2 says that $\mathcal{H}_{*}=\mathcal{H}_{\theta}$ up to a constant multiple of the norm, where $\mathcal{H}_{\theta}$ is the space defined in (1.7). In the guise of operator inequalities: for any fixed positive operators $A$ and $B$, the condition

$$
T^{*} T \leq M_{0} \quad, \quad T^{*} A T \leq M_{1} A \quad \Rightarrow \quad T^{*} B T \leq M_{0}{ }^{1-\theta} M_{1}{ }^{\theta} B
$$

is equivalent to that $B=A^{\theta}$.

It was observed in [27] that $\mathcal{H}_{\theta}$ also equals to the complex interpolation space $C_{\theta}(\overline{\mathcal{H}})$. For the sake of completeness, we supply a short proof of this fact in the appendix.

Remark 3.3. An exact quadratic interpolation method, the geometric mean was introduced earlier by Pusz and Woronowicz [33] (it corresponds to the $C_{1 / 2}$-method). In [40], Uhlmann generalized that method to a method (the quadratic mean) denoted $\mathrm{QI}_{t}$ where $0<t<1$; this method is quadratic and of exponent $t$.

In view of Theorem 3.2 and the preceding remarks we can conclude that $\mathrm{QI}_{\theta}(\overline{\mathcal{H}})=$ $C_{\theta}(\overline{\mathcal{H}})=\mathcal{H}_{\theta}$ for any regular Hilbert couple $\overline{\mathcal{H}}$. We refer to 40 for several physically relevant applications of this type of interpolation.

Finally, we want to mention that in 32] Peetre introduces the "Riesz method of interpolation"; in Section 5 he also defines a related method "QM" which comes close to the complex $C_{1 / 2}$-method.

3.3. Donoghue's theorem. The exact quadratic interpolation spaces relative to a Hilbert couple were characterized by Donoghue in the paper [13. We shall here prove the following equivalent version of Donoghue's result (see [2, 3]).

Theorem III. An intermediate Hilbert space $\mathcal{H}_{*}$ relative to $\overline{\mathcal{H}}$ is an exact interpolation space if and only if there is a positive radon measure $\varrho$ on $[0, \infty]$ such that

$$
\|x\|_{*}^{2}=\int_{[0, \infty]}\left(1+t^{-1}\right) K(t, x ; \overline{\mathcal{H}}) d \varrho(t) .
$$

Equivalently, $\mathcal{H}_{*}$ is exact interpolation relative to $\overline{\mathcal{H}}$ if and only if the corresponding operator $B$ can be represented as $B=h(A)$ for some function $h \in P^{\prime}$.

The statements that all norms of the given form are exact quadratic interpolation norms have already been shown (see 1.2 . There remains to prove that there are no others. 
Donoghue's original formulation of the result, as well as other equivalent forms of the theorem, is found in Section 6] below. Our present approach follows [2] and is based on $K$-monotonicity.

Remark 3.4. The condition that $\mathcal{H}_{*}$ be exact interpolation with respect to $\overline{\mathcal{H}}$ means that $\mathcal{H}_{*}$ is of type $\mathbf{H}$ where $\mathbf{H}(t)=\max \{1, t\}$. In view of Theorem $\Pi$ (and Remark 3.1), this means that we can represent $B=h(A)$ where $h$ is quasi-concave on $\sigma(A) \backslash\{0\}$,

$$
h(\lambda) \leq h(\mu) \max \{1, \lambda / \mu\}, \quad \lambda, \mu \in \sigma(A) \backslash\{0\} .
$$

In particular, $h$ is locally Lipschitzian on $\sigma(A) \cap \mathbf{R}_{+}$.

Remark 3.5. A related result concerning non-exact quadratic interpolation was proved by Ovchinnikov [30] using Donoghue's theorem. Cf. also [4].

3.4. The proof for simple finite-dimensional couples. Similar to our approach to Calderón's problem, our strategy is to reduce Theorem III to a case of "simple couples".

Theorem 3.6. Assume that $\mathcal{H}_{0}=\mathcal{H}_{0}=\mathbf{C}^{n}$ as sets and that all eigenvalues $\left(\lambda_{i}\right)_{1}^{n}$ of the corresponding operator $A$ are of unit multiplicity. Consider a third Hermitian norm $\|x\|_{*}^{2}=\langle B x, x\rangle_{0}$ on $\mathbf{C}^{n}$. Then $\mathcal{H}_{*}$ is exact interpolation with respect to $\overline{\mathcal{H}}$ if and only if $B=h(A)$ where $h \in P^{\prime}$.

Remark 3.7. The lemma says that the class of functions $h$ on $\sigma(A)$ satisfying

$$
T^{*} T \leq 1 \quad, \quad T^{*} A T \leq A \quad \Rightarrow \quad T^{*} h(A) T \leq h(A), \quad\left(T \in M_{n}(\mathbf{C})\right)
$$

is precisely the set $P^{\prime} \mid \sigma(A)$ of restrictions of $P^{\prime}$-functions to $\sigma(A)$. In this way, the condition (3.6) provides an operator-theoretic solution to the interpolation problem by positive Pick functions on a finite subset of $\mathbf{R}_{+}$.

Proof of Theorem [3.6. We already know that the spaces $\mathcal{H}_{*}$ of the asserted form are exact interpolation relative to $\overline{\mathcal{H}}$ (see subsections 1.2 and 1.3 ).

Now let $\mathcal{H}_{*}$ be any exact quadratic interpolation space. By Donoghue's lemma and the argument in 2.3 , we can for an appropriate positive sequence $\lambda=\left(\lambda_{i}\right)_{1}^{n}$ identify $\overline{\mathcal{H}}=\overline{\ell_{2}^{n}}(\lambda), A=\operatorname{diag}\left(\lambda_{i}\right)$, and $B=h(A)$ where $h$ is some positive function defined on $\sigma(A)=\left\{\lambda_{i}\right\}_{1}^{n}$.

Our assumption is that $\ell_{2}^{n}(h(\lambda))$ is exact interpolation relative to $\overline{\ell_{2}^{n}}(\lambda)$. We must prove that $h \in P^{\prime} \mid \sigma(A)$. To this end, write

$$
k_{\lambda_{i}}(t)=\frac{(1+t) \lambda_{i}}{1+t \lambda_{i}}
$$

and recall that (see Lemma 1.1)

$$
K\left(t, x ; \overline{\ell_{2}^{n}}(\lambda)\right)=\left(1+t^{-1}\right)^{-1} \sum_{1}^{n}\left|x_{i}\right|^{2} k_{\lambda_{i}}(t) .
$$

Let us denote by $C$ the algebra of continuous complex functions on $[0, \infty]$ with the supremum norm $\|u\|_{\infty}=\sup _{t>0}|u(t)|$. Let $V \subset C$ be the linear span of the $k_{\lambda_{i}}$ for $i=1, \ldots, n$. We define a positive functional $\phi$ on $V$ by

$$
\phi\left(\sum_{1}^{n} a_{i} k_{\lambda_{i}}\right)=\sum_{1}^{n} a_{i} h\left(\lambda_{i}\right) .
$$


We claim that $\phi$ is a positive functional, i.e., if $u \in V$ and $u(t) \geq 0$ for all $t>0$, then $\phi(u) \geq 0$.

To prove this let $u=\sum_{1}^{n} a_{i} k_{\lambda_{i}}$ be non-negative on $\mathbf{R}_{+}$and write $a_{i}=\left|x_{i}\right|^{2}-\left|y_{i}\right|^{2}$ for some $x, y \in \mathbf{C}^{n}$. The condition that $u \geq 0$ means that

$$
\begin{aligned}
\left(1+t^{-1}\right) K\left(t, x ; \overline{\ell_{2}^{n}}(\lambda)\right) & =\sum_{i=1}^{n}\left|x_{i}\right|^{2} k_{\lambda_{i}}(t) \\
& \geq \sum_{i=1}^{n}\left|y_{i}\right|^{2} k_{\lambda_{i}}(t) \\
& =\left(1+t^{-1}\right) K\left(t, y ; \overline{\ell_{2}^{n}}(\lambda)\right), \quad t>0 .
\end{aligned}
$$

Since $\overline{\ell_{2}^{n}}(\lambda)$ is an exact Calderón couple (by Theorem 2.4), the space $\ell_{2}^{n}(h(\lambda)$ ) is exact $K$-monotonic. In other words, (3.7) implies that

i.e.,

$$
\|x\|_{\ell_{2}^{n}(h(\lambda))} \geq\|y\|_{\ell_{2}^{n}(h(\lambda))},
$$

$$
\phi(u)=\sum_{1}^{n}\left(\left|x_{i}\right|^{2}-\left|y_{i}\right|^{2}\right) h\left(\lambda_{i}\right) \geq 0 .
$$

The asserted positivity of $\phi$ is thereby proved.

Replacing $\lambda_{i}$ by $c \lambda_{i}$ for a suitable positive constant $c$ we can without losing generality assume that $1 \in \sigma(A)$, i.e., that the unit $\mathbf{1}(x) \equiv 1$ of the $C^{*}$-algebra $C$ belongs to $V$. The positivity of $\phi$ then ensures that

$$
\|\phi\|=\sup _{u \in V ;\|u\|_{\infty} \leq 1}|\phi(u)|=\phi(\mathbf{1}) .
$$

Let $\Phi$ be a Hahn-Banach extension of $\phi$ to $C$ and note that

$$
\|\Phi\|=\|\phi\|=\phi(\mathbf{1})=\Phi(\mathbf{1}) .
$$

This means that $\Phi$ is a positive functional on $C$ (cf. [29], §3.3). By the Riesz representation theorem there is thus a positive Radon measure $\varrho$ on $[0, \infty]$ such that

In particular

$$
\Phi(u)=\int_{[0, \infty]} u(t) d \varrho(t), \quad u \in C .
$$

$$
h\left(\lambda_{i}\right)=\phi\left(k_{\lambda_{i}}\right)=\Phi\left(k_{\lambda_{i}}\right)=\int_{[0, \infty]} \frac{(1+t) \lambda_{i}}{1+t \lambda_{i}} d \varrho(t), \quad i=1, \ldots, n .
$$

We have shown that $h$ is the restriction to $\sigma(A)$ of a function of class $P^{\prime}$.

3.5. The proof of Donoghue's theorem. We here prove Theorem III in full generality.

We remind the reader that if $S \subset \mathbf{R}_{+}$is a subset, we write $P^{\prime} \mid S$ for the convex cone of restrictions of $P^{\prime}$-functions to $S$. We first collect some simple facts about this cone.

Lemma 3.8. (i) The class $P^{\prime} \mid S$ is closed under pointwise convergence.

(ii) If $S$ is finite and if $\lambda=\left(\lambda_{i}\right)_{i=1}^{n}$ is an enumeration of the points of $S$ then $h$ belongs to $P^{\prime} \mid S$ if and only if $\ell_{2}^{n}(h(\lambda))$ is exact interpolation with respect to the pair $\overline{\ell_{2}^{n}}(\lambda)$. 
(iii) If $S$ is infinite, then a continuous function $h$ on $S$ belongs to $P^{\prime} \mid S$ if and only if $h \in P^{\prime} \mid \Lambda$ for every finite subset $\Lambda \subset S$.

Proof. (i) Let $h_{n}$ be a sequence in $P^{\prime}$ converging pointwise on $S$ and fix $\lambda \in S$. It is clear that the boundedness of the numbers $h_{n}(\lambda)$ is equivalent to boundedness of the total masses of the corresponding measures $\varrho_{n}$ on the compact set $[0, \infty]$. It now suffices to apply Helly's selection theorem.

(ii) This is Theorem 3.6.

(iii) Let $\Lambda_{n}$ be an increasing sequence of finite subsets of $S$ whose union is dense. Let $h_{n}=h \mid \Lambda_{n}$ where $h$ is continuous on $S$. If $h_{n} \in P^{\prime} \mid \Lambda_{n}$ for all $n$ then the sequence $h_{n}$ converges pointwise on $\cup \Lambda_{n}$ to $h$. By part (i) we then have $h \in P^{\prime} \mid \sigma(A)$.

We can now finish the proof of Donoghue's theorem (Theorem III).

Let $\mathcal{H}_{*}$ be exact interpolation with respect to $\overline{\mathcal{H}}$ and represent the corresponding operator as $B=h(A)$ where $h$ satisfies (3.3). By the remarks after Theorem III] the function $h$ is locally Lipschitzian.

In view of Lemma 3.8 we shall be done when we have proved that $\ell_{2}^{n}(h(\lambda))$ is exact interpolation with respect to $\overline{\ell_{2}^{n}}(\lambda)$ for all sequences $\lambda=\left(\lambda_{i}\right)_{1}^{n} \subset \sigma(A)$ of distinct points. Let us arrange the sequences in increasing order: $0<\lambda_{1}<\cdots<\lambda_{n}$.

Fix $\varepsilon>0, \varepsilon<\min \left\{c, \lambda_{1}, 1 / \lambda_{n}\right\}$ and let $E_{i}=\left[\lambda_{i}-\varepsilon, \lambda_{i}+\varepsilon\right] \cap \sigma(A)$; we assume that $\varepsilon$ is sufficiently small that the $E_{i}$ be disjoint. Let $M=\cup_{1}^{n} E_{i}$. We can assume that $h$ has Lipschitz constant at most 1 on $M$.

Let $\mathcal{M}$ be the reducing subspace of $\mathcal{H}_{0}$ corresponding to the spectral set $M$, and let $\tilde{A}$ be the compression of $A$ to $\mathcal{M}$. We define a function $g$ on $M$ by $g(\lambda)=\lambda_{i}$ on $E_{i}$. Then $|g(\lambda)-\lambda|<\varepsilon$ on $\sigma(\tilde{A})$, so

$$
\|\tilde{A}-g(\tilde{A})\| \leq \varepsilon \quad, \quad\|h(\tilde{A})-h(g(\tilde{A}))\| \leq \varepsilon .
$$

Lemma 3.9. Suppose that $A^{\prime}, A^{\prime \prime} \in \mathcal{L}(\mathcal{M})$ satisfy $A^{\prime}, A^{\prime \prime} \geq \delta>0$ and $\left\|A^{\prime}-A^{\prime \prime}\right\| \leq$ $\varepsilon$. Then $\|T\|_{A^{\prime \prime}} \leq \sqrt{1+2 \varepsilon / \delta} \max \left\{\|T\|,\|T\|_{A^{\prime}}\right\}$ for all $T \in \mathcal{L}(\mathcal{M})$.

Proof. By definition, $\|T\|_{A^{\prime}}$ is the smallest number $C \geq 0$ such that $T^{*} A^{\prime} T \leq$ $C^{2} A^{\prime}$. Thus

$$
\begin{aligned}
T^{*} A^{\prime \prime} T & =T^{*}\left(A^{\prime \prime}-A^{\prime}\right) T+T^{*} A^{\prime} T \\
& \leq\|T\|^{2} \varepsilon+\|T\|_{A^{\prime}}^{2}\left(A^{\prime \prime}+\left(A^{\prime}-A^{\prime \prime}\right)\right) \\
& \leq 2 \varepsilon \max \left\{\|T\|^{2},\|T\|_{A^{\prime}}^{2}\right\}+\|T\|_{A^{\prime}}^{2} A^{\prime \prime} \\
& \leq \max \left\{\|T\|^{2},\|T\|_{A^{\prime}}^{2}\right\}(1+2 \varepsilon / \delta) A^{\prime \prime} .
\end{aligned}
$$

We can find $\delta>0$ such that the operators $\tilde{A}, g(\tilde{A}), h(\tilde{A})$, and $h(g(\tilde{A}))$ are $\geq \delta$. Then by repeated use of Lemma 3.9.

$$
\begin{aligned}
\|T\|_{h(g(\tilde{A}))} & \leq \sqrt{1+2 \varepsilon / \delta} \max \left\{\|T\|,\|T\|_{h(\tilde{A})}\right\} \\
& \leq \sqrt{1+2 \varepsilon / \delta} \max \left\{\|T\|,\|T\|_{\tilde{A}}\right\} \\
& \leq(1+2 \varepsilon / \delta) \max \left\{\|T\|,\|T\|_{g(\tilde{A})}\right\}, \quad T \in \mathcal{L}(\mathcal{M}) .
\end{aligned}
$$

Let $e_{i}$ be a unit vector supported by the spectral set $E_{i}$ and define a space $\mathcal{V} \subset \mathcal{M}$ to be the $n$-dimensional space spanned by the $e_{i}$. Let $A_{0}$ be the compression of 
$g(\tilde{A})$ to $\mathcal{V}$; then

$$
\|T\|_{h\left(A_{0}\right)} \leq(1+2 \varepsilon / \delta) \max \left\{\|T\|,\|T\|_{A_{0}}\right\}, \quad T \in \mathcal{L}(\mathcal{V}) .
$$

Identifying $\mathcal{V}$ with $\ell_{2}^{n}$ and $A_{0}$ with the matrix $\operatorname{diag}\left(\lambda_{i}\right)$, we see that (3.9) is independent of $\varepsilon$. Letting $\varepsilon$ diminish to 0 in (3.9) now gives that $\ell_{2}^{n}(h(\lambda))$ is exact interpolation with respect to $\overline{\ell_{2}^{n}}(\lambda)$. In view of Lemma 3.8, this finishes the proof of Theorem ऑII. q.e.d.

\section{Classes of matrix functions}

In this section, we discuss the basic properties of interpolation functions: in particular, the relation to the well known classes of monotone matrix functions. We refer to the books [12] and [34] for further reading on the latter classes.

4.1. Interpolation and matrix monotone functions. Let $A_{1}$ and $A_{2}$ be positive operators in $\ell_{2}^{n}\left(n=\infty\right.$ is admitted). Suppose that $A_{1} \leq A_{2}$ and form the following operators on $\ell_{2}^{n} \oplus \ell_{2}^{n}$,

$$
T_{0}=\left(\begin{array}{ll}
0 & 0 \\
1 & 0
\end{array}\right) \quad, \quad A=\left(\begin{array}{cc}
A_{2} & 0 \\
0 & A_{1}
\end{array}\right) .
$$

It is then easy to see that $T_{0}{ }^{*} T_{0} \leq 1$ and that $T_{0}{ }^{*} A T_{0}=\left(\begin{array}{cc}A_{1} & 0 \\ 0 & 0\end{array}\right) \leq A$.

Now assume that a function $h$ on $\sigma(A)$ belongs to the class $C_{A}$ defined in $\$ 1.4$, i.e., that $h$ satisfies

$$
T^{*} T \leq 1 \quad, \quad T^{*} A T \leq A \quad \Rightarrow \quad T^{*} h(A) T \leq h(A),
$$

where $T$ denotes an operator on $\ell_{2}^{2 n}$.

We then have $T_{0}{ }^{*} h(A) T_{0} \leq h(A)$, or

$$
\left(\begin{array}{cc}
h\left(A_{1}\right) & 0 \\
0 & 0
\end{array}\right) \leq\left(\begin{array}{cc}
h\left(A_{2}\right) & 0 \\
0 & h\left(A_{1}\right)
\end{array}\right) .
$$

In particular, we find that $h\left(A_{1}\right) \leq h\left(A_{2}\right)$. We have shown that (under the assumptions above)

$$
A_{1} \leq A_{2} \quad \Rightarrow \quad h\left(A_{1}\right) \leq h\left(A_{2}\right) .
$$

We now change our point of view slightly. Given a positive integer $n$, we let $C_{n}$ denote the convex of positive functions $h$ on $\mathbf{R}_{+}$such that (4.1) holds for all positive operators $A$ on $\ell_{2}^{n}$ and all $T \in \mathcal{L}\left(\ell_{2}^{n}\right)$.

Similarly, we let $P_{n}^{\prime}$ denote the class of all positive functions $h$ on $\mathbf{R}_{+}$such that $h\left(A_{1}\right) \leq h\left(A_{2}\right)$ whenever $A_{1}, A_{2}$ are positive operators on $\ell_{2}^{n}$ such that $A_{1} \leq A_{2}$. We refer to $P_{n}^{\prime}$ as the cone of positive functions monotone of order $n$ on $\mathbf{R}_{+}$.

We have shown above that $C_{2 n} \subset P_{n}^{\prime}$.

In the other direction, assume that $h \in P_{2 n}^{\prime}$. Let $A, T$ be bounded operators on $\ell_{2}^{n}$ with $A>0, T^{*} T \leq 1$ and $T^{*} A T \leq A$. Assume also that $h$ be continuous. We will use the following lemma due to Hansen [19]. We recall the proof for completeness.

Lemma 4.1. ([19]) $T^{*} h(A) T \leq h\left(T^{*} A T\right)$.

Proof. Put $S=\left(1-T T^{*}\right)^{1 / 2}$ and $R=\left(1-T^{*} T\right)^{1 / 2}$ and consider the $2 n \times 2 n$ matrix

$$
U=\left(\begin{array}{cc}
T & S \\
R & -T^{*}
\end{array}\right) \quad, \quad X=\left(\begin{array}{cc}
A & 0 \\
0 & 0
\end{array}\right) .
$$


It is well-known, and easy to check, that $U$ is unitary and that

$$
U^{*} X U=\left(\begin{array}{cc}
T^{*} A T & T^{*} A S \\
S A T & S A S
\end{array}\right)
$$

Next fix a number $\varepsilon>0$, a constant $\lambda>0$ (to be fixed), and form the matrix

$$
Y=\left(\begin{array}{cc}
T^{*} A T+\varepsilon & 0 \\
0 & 2 \lambda
\end{array}\right)
$$

which, provided that we choose $\lambda \geq\|S A S\|$, satisfies

$$
Y-U^{*} X U=\left(\begin{array}{cc}
\varepsilon & -T^{*} A S \\
-S A T & 2 \lambda-S A S
\end{array}\right) \geq\left(\begin{array}{cc}
\varepsilon & D \\
D^{*} & \lambda
\end{array}\right),
$$

where we have written $D=-T^{*} A S$.

If we now also choose $\lambda$ so that $\lambda \geq\|D\|^{2} / \varepsilon$, then we obtain for all $\xi, \eta \in \mathbf{C}^{n}$ that

$$
\begin{aligned}
\left\langle\left(\begin{array}{cc}
\varepsilon & D \\
D^{*} & \lambda
\end{array}\right)\left(\begin{array}{l}
\xi \\
\eta
\end{array}\right),\left(\begin{array}{l}
\xi \\
\eta
\end{array}\right)\right\rangle & =\varepsilon\|\xi\|^{2}+\langle D \eta, \xi\rangle+\left\langle D^{*} \xi, \eta\right\rangle+\lambda\|\eta\|^{2} \\
& \geq \varepsilon\|\xi\|^{2}-2\|D\|\|\xi\|\|\eta\|+\lambda\|\eta\|^{2} \geq 0 .
\end{aligned}
$$

Hence $U^{*} X U \leq Y$ and as a consequence $U^{*} h(X) U=h\left(U^{*} X U\right) \leq h(Y)$, since $h$ is matrix monotone of order $2 n$. The last inequality means that

$$
\left(\begin{array}{cc}
T^{*} h(A) T & T^{*} h(A) S \\
\operatorname{Sh}(A) T & S h(A) S
\end{array}\right) \leq\left(\begin{array}{cc}
h\left(T^{*} A T+\varepsilon\right) & 0 \\
0 & h(2 \lambda)
\end{array}\right),
$$

so in particular $T^{*} h(A) T \leq h\left(T^{*} A T+\varepsilon\right)$. Since $\varepsilon>0$ was arbitrary, and since $h$ is assumed to be continuous, we conclude the lemma.

We now continue our discussion. Assuming that $T^{*} T \leq 1$ and $T^{*} A T \leq A$, and that $h \in P_{2 n}^{\prime}$ is continuous, we have $h\left(T^{*} A T\right) \leq h(A)$ [since $h \in P_{n}^{\prime}$ ], so $T^{*} h(A) T \leq h(A)$ by Lemma 4.1. We conclude that $h \in C_{n}$.

To prove that $P_{2 n}^{\prime} \subset C_{n}$, we need to remove the continuity assumption on $h$ made above. This is completely standard: let $\varphi$ be a smooth positive function on $\mathbf{R}_{+}$such that $\int_{0}^{\infty} \varphi(t) d t / t=1$, and define a sequence $h_{k}$ by $h_{k}(\lambda)=$ $k^{-1} \int_{0}^{\infty} \varphi\left(\lambda^{k} / t^{k}\right) h(t) d t / t$. The class $P_{2 n}^{\prime}$ is a convex cone, closed under pointwise convergence [12], so the functions $h_{1}, h_{2}, \ldots$ are of class $P_{2 n}^{\prime}$. They are furthermore continuous, so by the argument above, they are of class $C_{n}$. By Lemma 3.8 the cone $C_{n}$ is also closed under pointwise convergence, so $h=\lim h_{n} \in C_{n}$.

To summarize, we have the inclusions $C_{2 n} \subset P_{n}^{\prime}, P_{2 n}^{\prime} \subset C_{n}$, and also $C_{n+1} \subset C_{n}$, $P_{n+1}^{\prime} \subset P_{n}^{\prime}$. In view of Theorem [II] we have the identity $\cap_{1}^{\infty} C_{n}=P^{\prime}$. The inclusions above now imply the following result, sometimes known as "Löwner's theorem on matrix monotone functions".

Theorem 4.2. We have $\cap_{1}^{\infty} P_{n}^{\prime}=\cap_{1}^{\infty} C_{n}=P^{\prime}$.

The identity $\cap_{1}^{\infty} P_{n}^{\prime}=P^{\prime}$ says that a positive function $h$ is monotone of all finite orders if and only it is of class $P^{\prime}$. The somewhat less precise fact that $P_{\infty}^{\prime}=P^{\prime}$ is interpreted as that the class of operator monotone functions coincides with $P^{\prime}$.

The identity $C_{\infty}=P^{\prime}$ is, except for notation, contained in the work of Foiaş and Lions, from [17. See $\$ 6.4$.

Note that the inclusion $P_{2 n}^{\prime} \subset C_{n}$ shows that a matrix monotone functions of order $2 n$ can be interpolated by a positive Pick function at $n$ points. Results of a 
similar nature, where it is shown, in addition, that an interpolating Pick function can be taken rational of a certain degree, are discussed, for example, in Donoghue's book [13, Chapter XIII] or (more relevant in the present connection) in the paper 14.

It seems somewhat inaccurate to refer to the identity $\cap_{1}^{\infty} P_{n}^{\prime}=P^{\prime}$ as "Löwner's theorem", since Löwner discusses more subtle results concerning matrix monotone functions of a given finite order $n$. In spite of this, it is common nowadays to let "Löwner's theorem" refer to this identity.

4.2. More on the cone $C_{A}$. We can now give an short proof of the following result due to Donoghue [14.

Theorem 4.3. For a positive function $h$ on $\sigma(A)$ we define two positive functions $\tilde{h}$ and $h^{*}$ on $\sigma\left(A^{-1}\right)$ by $\tilde{h}(\lambda)=\lambda h(1 / \lambda)$ and $h^{*}(\lambda)=1 / h(1 / \lambda)$. Then the following conditions are equivalent,

(i) $h \in C_{A}$,

(ii) $\tilde{h} \in C_{A^{-1}}$,

(iii) $h^{*} \in C_{A^{-1}}$.

Proof. Let $\mathcal{H}_{*}$ be a quadratic intermediate space relative to a regular Hilbert couple $\overline{\mathcal{H}}$; let $B=h(A)$ be the corresponding operator. It is clear that $\mathcal{H}_{*}$ is exact interpolation relative to $\overline{\mathcal{H}}$ if and only if $\mathcal{H}_{*}$ is exact interpolation relative to the reverse couple $\overline{\mathcal{H}^{(r)}}=\left(\mathcal{H}_{1}, \mathcal{H}_{0}\right)$. The latter couple has corresponding operator $A^{-1}$ and it is clear that the identity $\|x\|_{*}^{2}=\langle h(A) x, x\rangle_{0}$ is equivalent to that $\|x\|_{*}^{2}=\left\langle A^{-1} \tilde{h}\left(A^{-1}\right) x, x\right\rangle_{1}$. We have shown the equivalence of (i) and (ii).

Next let $\overline{\mathcal{H}^{*}}=\left(\mathcal{H}_{0}{ }^{*}, \mathcal{H}_{1}{ }^{*}\right)$ be the dual couple, where we identify $\mathcal{H}_{0}{ }^{*}=\mathcal{H}_{0}$. With this identification, $\mathcal{H}_{1}{ }^{*}$ becomes associated with the norm $\|x\|_{\mathcal{H}_{1}^{*}}^{2}=\left\langle A^{-1} x, x\right\rangle_{0}$, and $\mathcal{H}_{*}{ }^{*}$ is associated with $\|x\|_{\mathcal{H}_{*}^{*}}^{2}=\left\langle B^{-1} x, x\right\rangle_{0}$. It remains to note that $\mathcal{H}_{*}$ is exact interpolation relative to $\overline{\mathcal{H}}$ if and only if $\mathcal{H}_{*}{ }^{*}$ is exact interpolation relative to $\overline{\mathcal{H}^{*}}$, proving the equivalence of (i) and (iii).

Combining with Theorem III, one obtains alternative proofs of the interpolation theorems for $P^{\prime}$-functions discussed by Donoghue in the paper [14].

Remark 4.4. The exact quadratic interpolation spaces which are fixed by the duality, i.e., which satisfy $\mathcal{H}_{*}{ }^{*}=\mathcal{H}_{*}$, correspond precisely to the class of $P^{\prime}$-functions which are self-dual: $h^{*}=h$. This class was characterized by Hansen in the paper [20.

4.3. Matrix concavity. A function $h$ on $\mathbf{R}_{+}$is called matrix concave of order $n$ if we have Jensen's inequality

$$
\lambda h\left(A_{1}\right)+(1-\lambda) h\left(A_{2}\right) \leq h\left(\lambda A_{1}+(1-\lambda) A_{2}\right)
$$

for all positive $n \times n$ matrices $A_{1}, A_{2}$, and all numbers $\lambda \in[0,1]$. Let us denote by $\Gamma_{n}$ the convex cone of positive concave functions of order $n$ on $\mathbf{R}_{+}$. The fact that $\cap_{n} \Gamma_{n}=P^{\prime}$ follows from the theorem of Kraus [23]. Following [2] we now give an alternative proof of this fact.

Proposition 4.5. For all $n$ we have the inclusion $C_{3 n} \subset \Gamma_{n} \subset P_{n}^{\prime}$. In particular $\cap_{1}^{\infty} \Gamma_{n}=P^{\prime}$. 
Proof. Assume first that $h \in C_{3 n}$ and pick two positive matrices $A_{1}$ and $A_{2}$. Define $A_{3}=(1-\lambda) A_{1}+\lambda A_{2}$ where $\lambda \in[0,1]$ is given, and define matrices $A$ and $T$ of order $3 n$ by

$$
A=\left(\begin{array}{ccc}
A_{3} & 0 & 0 \\
0 & A_{1} & 0 \\
0 & 0 & A_{2}
\end{array}\right) \quad, \quad T=\left(\begin{array}{ccc}
0 & 0 & 0 \\
\sqrt{1-\lambda} & 0 & 0 \\
\sqrt{\lambda} & 0 & 0
\end{array}\right) .
$$

It is clear that $T^{*} T \leq 1$ and

$$
T^{*} A T=\left(\begin{array}{ccc}
A_{3} & 0 & 0 \\
0 & 0 & 0 \\
0 & 0 & 0
\end{array}\right) \leq A
$$

so, since $h \in C_{3 n}$, we have $T^{*} h(A) T \leq h(A)$, or

$$
\left(\begin{array}{ccc}
(1-\lambda) h\left(A_{1}\right)+\lambda h\left(A_{2}\right) & 0 & 0 \\
0 & 0 & 0 \\
0 & 0 & 0
\end{array}\right) \leq\left(\begin{array}{ccc}
h\left(A_{3}\right) & 0 & 0 \\
0 & h\left(A_{1}\right) & 0 \\
0 & 0 & h\left(A_{2}\right)
\end{array}\right) .
$$

Comparing the matrices in the upper left corners, we find that $h \in \Gamma_{n}$.

Assume now that $h \in \Gamma_{n}$, and take positive definite matrices $A_{1}, A_{2}$ of order $n$ with $A_{1} \leq A_{2}$. Also pick $\lambda \in(0,1)$. Then $\lambda A_{2}=\lambda A_{1}+(1-\lambda) A_{3}$ where $A_{3}=\lambda(1-\lambda)^{-1}\left(A_{2}-A_{1}\right)$. By matrix concavity, we then have

$$
h\left(\lambda A_{2}\right) \geq \lambda h\left(A_{1}\right)+(1-\lambda) h\left(A_{3}\right) \geq \lambda h\left(A_{1}\right),
$$

where we used non-negativity to deduce the last inequality. Being concave, $h$ is certainly continuous. Letting $\lambda \uparrow 1$ one thus finds that $h\left(A_{1}\right) \leq h\left(A_{2}\right)$. We have shown that $h \in P_{n}^{\prime}$.

For a further discussion of classes of convex matrix functions and their relations to monotonicity, we refer to the paper [21].

4.4. Interpolation functions of two variables. In this section, we briefly discuss a class of interpolation functions of two matrix variables. We shall not completely characterize the class of such generalized interpolation functions here, but we hope that the following discussion will be of some use for a future investigation.

Let $H_{1}$ and $H_{2}$ be Hilbert spaces. We turn $H_{1} \otimes H_{2}$ into a Hilbert space by defining the inner product on elementary tensors via $\left\langle x_{1} \otimes x_{2}, x_{1}^{\prime} \otimes x_{2}^{\prime}\right\rangle:=$ $\left\langle x_{1}, x_{1}{ }^{\prime}\right\rangle_{1} \cdot\left\langle x_{2}, x_{2}{ }^{\prime}\right\rangle_{2}$ (then extend via sesqui-linearity). Similarly, if $T_{i}$ are operators on $H_{i}$, the tensor product $T_{1} \otimes T_{2}$ is defined on elementary tensors via $\left(T_{1} \otimes T_{2}\right)\left(x_{1} \otimes x_{2}\right)=T_{1} x_{1} \otimes T_{2} x_{2}$. It is then easy to see that if $A_{i}$ are positive operators on $H_{i}$ for $i=1,2$, then $A_{1} \otimes A_{2} \geq 0$ as an operator on the tensor product. Furthermore, we have $A_{1} \otimes A_{2} \leq A_{1}^{\prime} \otimes A_{2}^{\prime}$ if $A_{i} \leq A_{i}^{\prime}$ for $i=1,2$.

Given two positive definite matrices $A_{i}$ of orders $n_{i}$ and a function $h$ on $\sigma\left(A_{1}\right) \times$ $\sigma\left(A_{2}\right)$, we define a matrix $h\left(A_{1}, A_{2}\right)$ by

$$
h\left(A_{1}, A_{2}\right)=\sum_{\left(\lambda_{1}, \lambda_{2}\right) \in \sigma\left(A_{1}\right) \times \sigma\left(A_{2}\right)} h\left(\lambda_{1}, \lambda_{2}\right) E_{\lambda_{1}}^{1} \otimes E_{\lambda_{2}}^{2}
$$

where $E^{j}$ is the spectral resolution of the matrix $A_{j}$.

We shall say that $h$ gives rise to exact interpolation relative to $\left(A_{1}, A_{2}\right)$, and write $h \in C_{A_{1}, A_{2}}$, if the condition

$$
T_{j}{ }^{*} T_{j} \leq 1 \quad, \quad T_{j}{ }^{*} A_{j} T_{j} \leq A_{j}, \quad j=1,2
$$


implies

$$
\begin{aligned}
h\left(A_{1}, A_{2}\right) & +\left(T_{1} \otimes T_{2}\right)^{*} h\left(A_{1}, A_{2}\right)\left(T_{1} \otimes T_{2}\right) \\
& -\left(T_{1} \otimes 1\right)^{*} h\left(A_{1}, A_{2}\right)\left(T_{1} \otimes 1\right)-\left(1 \otimes T_{2}\right)^{*} h\left(A_{1}, A_{2}\right)\left(1 \otimes T_{2}\right) \geq 0 .
\end{aligned}
$$

Taking $T_{1}=T_{2}=0$ we see that $h \geq 0$ for all $h \in C_{A_{1}, A_{2}}$. It is also clear that $C_{A_{1}, A_{2}}$ is a convex cone closed under pointwise convergence on the finite set $\sigma\left(A_{1}\right) \times \sigma\left(A_{2}\right)$.

If $h=h_{1} \otimes h_{2}$ is an elementary tensor where $h_{j} \in C_{A_{j}}$ is a function of one variable, then (4.3) implies $T_{j}{ }^{*} h_{j}\left(A_{j}\right) T_{j} \leq h_{j}\left(A_{j}\right)$, whence $\left(h_{1}\left(A_{1}\right)-T_{1}{ }^{*} h_{1}\left(A_{1}\right) T_{1}\right) \otimes$ $\left(h_{2}\left(A_{2}\right)-T_{2}{ }^{*} h_{2}\left(A_{2}\right) T_{2}\right) \geq 0$, which implies (4.4). We have shown that $C_{A_{1}} \otimes C_{A_{2}} \subset$ $C_{A_{1}, A_{2}}$.

Since for each $t \geq 0$ the $P^{\prime}$-function $\lambda \mapsto \frac{(1+t) \lambda}{1+t \lambda}$ is of class $C_{A_{j}}$, we infer that every function representable in the form

$$
h\left(\lambda_{1}, \lambda_{2}\right)=\iint_{[0, \infty]^{2}} \frac{\left(1+t_{1}\right) \lambda_{1}}{1+t_{1} \lambda_{1}} \frac{\left(1+t_{2}\right) \lambda_{2}}{1+t_{2} \lambda_{2}} d \varrho\left(t_{1}, t_{2}\right)
$$

with some positive Radon measure $\varrho$ on $[0, \infty]^{2}$ is in the class $C_{A_{1}, A_{2}}$.

We shall say that a function $h$ on $\sigma\left(A_{1}\right) \times \sigma\left(A_{2}\right)$ has the separate interpolationproperty if for each fixed $b \in \sigma\left(A_{2}\right)$ the function $\lambda_{1} \mapsto h\left(\lambda_{1}, b\right)$ is of class $C_{A_{1}}$, and a similar statement holds for all functions $\lambda_{2} \mapsto h\left(a, \lambda_{2}\right)$.

Lemma 4.6. Each function of class $C_{A_{1}, A_{2}}$ has the separate interpolation-property.

Proof. Let $T_{2}=0$ and take an arbitrary $T_{1}$ with $T_{1}{ }^{*} T_{1} \leq 1$ and $T_{1}{ }^{*} A_{1} T_{1} \leq A_{1}$. By hypothesis,

$$
\left(T_{1} \otimes 1\right)^{*} h\left(A_{1}, A_{2}\right)\left(T_{1} \otimes 1\right) \leq h\left(A_{1}, A_{2}\right) .
$$

Fix an eigenvalue $b$ of $A_{2}$ and let $y$ be a corresponding normalized eigenvector. Then for all $x \in H_{1}$ we have $\left\langle h\left(A_{1}, A_{2}\right) x \otimes y, x \otimes y\right\rangle=\left\langle h\left(A_{1}, b\right) x, x\right\rangle_{H_{1}}$ and $\left\langle\left(T_{1} \otimes 1\right)^{*} h\left(A_{1}, A_{2}\right)\left(T_{1} \otimes 1\right) x \otimes y, x \otimes y\right\rangle=\left\langle T_{1}{ }^{*} h\left(A_{1}, b\right) T_{1} x, x\right\rangle_{H_{1}}$ so

$$
\left\langle T_{1}{ }^{*} h\left(A_{1}, b\right) T_{1} x, x\right\rangle_{H_{1}} \leq\left\langle h\left(A_{1}, b\right) x, x\right\rangle_{H_{1}} .
$$

The functions $h\left(a, \lambda_{2}\right)$ can be treated similarly.

Example. The function $h\left(\lambda_{1}, \lambda_{2}\right)=\left(\lambda_{1}+\lambda_{2}\right)^{1 / 2}$ clearly has the separate interpolationproperty for all $A_{1}, A_{2}$. However, it is not representable in the form (4.5). Indeed, $\operatorname{Re}\{h(i, i)-h(-i, i)\}=1$ while it is easy to check that $\operatorname{Re}\left\{h\left(\lambda_{1}, \lambda_{2}\right)-h\left(\bar{\lambda}_{1}, \lambda_{2}\right)\right\} \leq 0$ whenever $\operatorname{Im} \lambda_{1}, \operatorname{Im} \lambda_{2}>0$ and $h$ is of the form (4.5).

Let us say that a function $h\left(\lambda_{1}, \lambda_{2}\right)$ defined on $\mathbf{R}_{+} \times \mathbf{R}_{+}$is an interpolation function (of two variables) if $h \in C_{A_{1}, A_{2}}$ for all $A_{1}, A_{2}$. Lemma 4.6 implies that interpolation functions are separately real-analytic in $\mathbf{R}_{+} \times \mathbf{R}_{+}$and that the functions $h(a, \cdot)$ and $h(\cdot, b)$ are of class $P^{\prime}$ (cf. Theorem III).

The above notion of interpolation function is close to Korányi's definition of monotone matrix function of two variables: $f\left(\lambda_{1}, \lambda_{2}\right)$ is matrix monotone in a rectangle $I=I_{1} \times I_{2}\left(I_{1}, I_{2}\right.$ intervals in $\left.\mathbf{R}\right)$ if $A_{1} \leq A_{1}^{\prime}$ (with spectra in $\left.I_{1}\right)$ and $A_{2} \leq A_{2}^{\prime}$ (with spectra in $I_{2}$ ) implies

$$
f\left(A_{1}^{\prime}, A_{2}^{\prime}\right)-f\left(A_{1}^{\prime}, A_{2}\right)-f\left(A_{1}, A_{2}^{\prime}\right)+f\left(A_{1}, A_{2}\right) \geq 0 .
$$

Lemma 4.7. Each interpolation function is matrix monotone in $\mathbf{R}_{+} \times \mathbf{R}_{+}$. 
Proof. Let $0<A_{i} \leq A_{i}^{\prime}$ and put $\tilde{A}_{i}=\left(\begin{array}{cc}A_{i}^{\prime} & 0 \\ 0 & A_{i}\end{array}\right), T_{i}=\left(\begin{array}{ll}0 & 0 \\ 1 & 0\end{array}\right)$. Since $T_{i}^{*} \tilde{A}_{i} T_{i} \leq$ $\tilde{A}_{i}$, an interpolation function $h$ will satisfy the interpolation inequality (4.4) with $A_{i}$ replaced by $\tilde{A}_{i}$. Applying this inequality to vectors of the form $\left(\begin{array}{c}x_{1} \\ 0\end{array}\right) \otimes\left(\begin{array}{c}x_{2} \\ 0\end{array}\right)$ we readily obtain

$$
\begin{aligned}
& \left\langle h\left(A_{1}^{\prime}, A_{2}^{\prime}\right) x_{1} \otimes x_{2}, x_{1} \otimes x_{2}\right\rangle-\left\langle h\left(A_{1}, A_{2}^{\prime}\right) x_{1} \otimes x_{2}, x_{1} \otimes x_{2}\right\rangle \\
& -\left\langle h\left(A_{1}^{\prime}, A_{2}\right) x_{1} \otimes x_{2}, x_{1} \otimes x_{2}\right\rangle+\left\langle h\left(A_{1}, A_{2}\right) x_{1} \otimes x_{2}, x_{1} \otimes x_{2}\right\rangle \geq 0 .
\end{aligned}
$$

The same result obtains with $x_{1} \otimes x_{2}$ replaced by a sum $x_{1} \otimes x_{2}+x_{1}^{\prime} \otimes x_{2}^{\prime}+\ldots$, i.e., $h$ is matrix monotone.

Remark 4.8. Assume that $f$ is of the form $f\left(\lambda_{1}, \lambda_{2}\right)=g_{1}\left(\lambda_{1}\right)+g_{2}\left(\lambda_{2}\right)$. Then $f$ is matrix monotone for all $g_{1}, g_{2}$ and $f$ is an interpolation function if and only if $g_{1}, g_{2} \in P^{\prime}$. In order to disregard "trivial" monotone functions of the above type, Korányi [22] imposed the normalizing assumption (a) $f\left(\lambda_{1}, 0\right)=f\left(0, \lambda_{2}\right)=0$ for all $\lambda_{1}, \lambda_{2}$.

It follows from Lemma 4.7 and the proof of [22, Theorem 4] that, if $h$ is a $C^{2}$-smooth interpolation function, then the function

$$
k\left(x_{1}, x_{2} ; y_{1}, y_{2}\right)=\frac{h\left(x_{1}, x_{2}\right)-h\left(x_{1}, y_{2}\right)-h\left(y_{1}, x_{2}\right)+h\left(y_{1}, y_{2}\right)}{\left(x_{1}-y_{1}\right)\left(x_{2}-y_{2}\right)}
$$

is positive definite in the sense that $\sum_{m} \sum_{n} k\left(x_{m}, y_{m} ; x_{n}, y_{n}\right) \alpha_{m} \bar{\alpha}_{n} \geq 0$ for all finite sequences of positive numbers $x_{j}, y_{k}$ and all complex numbers $\alpha_{l}$. (The proof uses Löwner's matrix.) Korányi uses essentially this positive definiteness condition (and condition (a) in the remark above) to deduce an integral representation formula for $h$ as an integral of products of Pick functions. See Theorem 3 in [22]. However, in contrast to our situation, Korányi considers functions monotone on the rectangle $(-1,1) \times(-1,1)$, so this last result cannot be immediately applied. (It easily implies local representation formulas, valid in finite rectangles, but these representations do not appear to be very natural from our point of view.)

This is not the right place to attempt to extend Korányi's methods to functions on $\mathbf{R}_{+} \times \mathbf{R}_{+}$; it would seem more appropriate to give a more direct characterization of the classes $C_{A_{1}, A_{2}}$ or of the class of interpolation functions. At present, we do not know if there is an interpolation function which is not representable in the form (4.5).

\section{Proof of the $K$-Property}

In this section we extend the result of Theorem 2.4 to obtain the full proof of Theorem [1. The discussion is in principle not hard, but it does require some care to keep track of both norms when reducing to a finite-dimensional case.

Recall first that, by Lemma 2.3, it suffices to consider the diagonal case $\overline{\mathcal{H}}=\overline{\mathcal{K}}$.

To prove Theorem $\llbracket$ we fix a regular Hilbert couple $\overline{\mathcal{H}}$; we must prove that it has the $K$-property (see $\$ 1.5$ ). By Theorem 2.4, we know that this is true if $\overline{\mathcal{H}}$ is finite dimensional and the associated operator only has eigenvalues of unit multiplicity.

We shall use a weak ${ }^{*}$ type compactness result $([2])$. To formulate it, let $\mathcal{L}_{1}(\overline{\mathcal{H}})$ be the unit ball in the space $\mathcal{L}(\overline{\mathcal{H}})$. Moreover, let $\Sigma_{t}$ be the sum $\mathcal{H}_{0}+\mathcal{H}_{1}$ normed 
by $\|x\|_{\Sigma_{t}}^{2}:=K(t, x)$. Note that $\|\cdot\|_{\Sigma_{t}}$ is an equivalent norm on $\Sigma$ and that $\Sigma_{1}=\Sigma$ isometrically. We denote by $\mathcal{L}_{1}\left(\Sigma_{t}\right)$ the unit ball in the space $\mathcal{L}\left(\Sigma_{t}\right)$.

In view of Remark 1.4 one has the identity

$$
\mathcal{L}_{1}(\overline{\mathcal{H}})=\bigcap_{t \in \mathbf{R}_{+}} \mathcal{L}_{1}\left(\Sigma_{t}\right)
$$

We shall use this to define a compact topology on $\mathcal{L}_{1}(\overline{\mathcal{H}})$.

Lemma 5.1. The subset $\mathcal{L}_{1}(\overline{\mathcal{H}}) \subset \mathcal{L}_{1}(\Sigma)$ is compact relative to the weak operator topology inherited from $\mathcal{L}(\Sigma)$.

Recall that the weak operator topology on $\mathcal{L}(H)$ is the weakest topology such that a net $T_{i}$ converges to the limit $T$ if the inner product $\left\langle T_{i} x, y\right\rangle_{H}$ converges to $\langle T x, y\rangle_{H}$ for all $x, y \in H$.

Proof of Lemma 5.1. The weak operator topology coincides on the unit ball $\mathcal{L}_{1}(\Sigma)$ with the weak*-topology, which is compact, due to Alaoglu's theorem (see [29], Chap. 4 for details). It is clear that for a fixed $t>0$, the subset $\mathcal{L}_{1}(\Sigma) \cap \mathcal{L}_{1}\left(\Sigma_{t}\right)$ is weak operator closed in $\mathcal{L}_{1}(\Sigma)$; hence it is also compact. In view of (5.1), the set $\mathcal{L}_{1}(\overline{\mathcal{H}})$ is an intersection of compact sets. Hence the set $\mathcal{L}_{1}(\overline{\mathcal{H}})$ is itself compact, provided that we endow it with the subspace topology inherited from $\mathcal{L}_{1}(\Sigma)$.

Denote by $P_{n}$ the projections $P_{n}=E_{\sigma(A) \cap\left[n^{-1}, n\right]}$ on $\mathcal{H}_{0}$ where $E$ is the spectral resolution of $A$ and $n=1,2,3, \ldots$. Consider the couple

$$
\overline{\mathcal{H}^{(n)}}=\left(P_{n}\left(\mathcal{H}_{0}\right), P_{n}\left(\mathcal{H}_{1}\right)\right),
$$

the associated operator of which is the compression $A_{n}$ of $A$ to the subspace $P_{n}\left(\mathcal{H}_{0}\right)$. Note that the norms in the couple $\overline{\mathcal{H}^{(n)}}$ are equivalent, i.e., the associated operator $A_{n}$ is bounded above and below.

We shall need two lemmas.

Lemma 5.2. If $\overline{\mathcal{H}^{(n)}}$ has the $K$-property for all $n$, then so does $\overline{\mathcal{H}}$.

Proof. Note that $\left\|P_{n}\right\|_{\mathcal{L}(\overline{\mathcal{H}})}=1$ for all $n$, and that $P_{n} \rightarrow 1$ as $n \rightarrow \infty$ relative to the strong operator topology on $\mathcal{L}(\Sigma)$. Suppose that $x^{0}, y^{0} \in \Sigma$ are elements such that, for some $\rho>1$,

$$
K\left(t, y^{0}\right)<\frac{1}{\rho} K\left(t, x^{0}\right), \quad t>0 .
$$

Then $K\left(t, P_{n} y^{0}\right) \leq K\left(t, y^{0}\right)<\rho^{-1} K\left(t, x^{0}\right)$. Moreover, the identity $K\left(t, P_{n} y^{0}\right)=$ $\left\langle\frac{t A_{n}}{1+t A_{n}} P_{n} y^{0}, P_{n} y^{0}\right\rangle_{0}$ shows that we have an estimate of the form $K\left(t, P_{n} y^{0}\right) \leq$ $C_{n} \min \{1, t\}$ for $t>0$ and large enough $C_{n}$ (this follows since $A_{n}$ is bounded above and below).

The functions $K\left(t, P_{m} x^{0}\right)$ increase monotonically, converging uniformly on compact subsets of $\mathbf{R}_{+}$to $K\left(t, x^{0}\right)$ when $m \rightarrow \infty$. By concavity of the function $t \mapsto K\left(t, P_{m} x^{0}\right)$ we will then have

$$
K\left(t, P_{n} y^{0}\right)<\frac{1}{\tilde{\rho}} K\left(t, P_{m} x^{0}\right), \quad t \in \mathbf{R}_{+},
$$

provided that $m$ is sufficiently large, where $\tilde{\rho}$ is any number in the interval $1<\tilde{\rho}<\rho$. 
Indeed, let $A=\lim _{t \rightarrow \infty} K\left(t, P_{n} y^{0}\right)$ and $B=\lim _{t \rightarrow 0} K\left(t, P_{n} y^{0}\right) / t$. Take points $t_{0}<t_{1}$ such that $K\left(t, P_{n} y^{0}\right) \geq A / \rho^{\prime}$ when $t \geq t_{1}$ and $K\left(t, P_{n} y^{0}\right) / t \leq B \rho^{\prime}$ when $t \leq t_{0}$. Here $\rho^{\prime}$ is some number in the interval $1<\rho^{\prime}<\rho$.

Next use (5.2) to choose $m$ large enough that $K\left(t, P_{m} x^{0}\right)>\rho K\left(t, P_{n} y^{0}\right)$ for all $t \in\left[t_{0}, t_{1}\right]$. Then $K\left(t, P_{m} x^{0}\right)>\left(\rho / \rho^{\prime}\right) K\left(t, P_{n} y^{0}\right)$ for $t=t_{1}$, hence for all $t \geq t_{1}$, and $K\left(t, P_{m} x^{0}\right) / t>\left(\rho / \rho^{\prime}\right) K\left(t, P_{n} y^{0}\right) / t$ for $t=t_{0}$ and hence also when $t \leq t_{0}$. Choosing $\rho^{\prime}=\rho / \tilde{\rho}$ now establishes (5.3).

Put $N=\max \{m, n\}$. If $\overline{\mathcal{H}^{(N)}}$ has the $K$-property, we can find a map $T_{n m} \in$ $\mathcal{L}_{1}(\overline{\mathcal{H}})$ such that $T_{n m} P_{m} x^{0}=P_{n} y^{0}$. (Define $T_{m n}=0$ on the orthogonal complement of $P_{N}\left(\mathcal{H}_{0}\right)$ in $\Sigma$.) In view of Lemma 5.1 the maps $T_{n m}$ must cluster at some point $T \in \mathcal{L}_{1}(\overline{\mathcal{H}})$. It is clear that $T x^{0}=y^{0}$. Since $\rho>1$ was arbitrary, we have shown that $\overline{\mathcal{H}}$ has the $K$-property .

Lemma 5.3. Given $x^{0}, y^{0} \in \mathcal{H}_{0}^{(n)}$ and a number $\epsilon>0$ there exists a positive integer $n$ and a finite-dimensional couple $\overline{\mathcal{V}} \subset \overline{\mathcal{H}^{(n)}}$ such that $x^{0}, y^{0} \in \mathcal{V}_{0}+\mathcal{V}_{1}$ and

$$
(1-\epsilon) K(t, x ; \overline{\mathcal{H}}) \leq K(t, x ; \overline{\mathcal{V}}) \leq(1+\epsilon) K(t, x ; \overline{\mathcal{H}}), \quad t>0, x \in \mathcal{V}_{0}+\mathcal{V}_{1}
$$

Moreover, $\overline{\mathcal{V}}$ can be chosen so that all eigenvalues of the associated operator $A_{\overline{\mathcal{V}}}$ are of unit multiplicity.

Proof. Let $A_{n}$ be the operator associated with the couple $\overline{\mathcal{H}^{(n)}}$; thus $1 / n \leq A_{n} \leq n$.

Take $\eta>0$ and let $\left\{\lambda_{i}\right\}_{1}^{N}$ be a finite subset of $\sigma\left(A_{n}\right)$ such that $\sigma\left(A_{n}\right) \subset \cup_{1}^{N} E_{i}$ where $E_{i}=\left(\lambda_{i}-\eta / 2, \lambda_{i}+\eta / 2\right)$. We define a Borel function $w: \sigma\left(A_{n}\right) \rightarrow \sigma\left(A_{n}\right)$ by $w(\lambda)=\lambda_{i}$ on $E_{i} \cap \sigma\left(A_{n}\right)$; then $\left\|w\left(A_{n}\right)-A_{n}\right\|_{\mathcal{L}\left(\mathcal{H}_{0}\right)} \leq \eta$.

Let $k_{t}(\lambda)=\frac{t \lambda}{1+t \lambda}$. It is easy to check that the Lipschitz constant of the restriction $k_{t} \mid \sigma\left(A_{n}\right)$ is bounded above by $C_{1} \min \{1, t\}$ where $C_{1}=C_{1}(n)$ is independent of $t$. Hence

$$
\left\|k_{t}\left(w\left(A_{n}\right)\right)-k_{t}\left(A_{n}\right)\right\|_{\mathcal{L}\left(\mathcal{H}_{0}\right)} \leq C_{1} \eta \min \{1, t\} .
$$

It follows readily that

$$
\left|\left\langle\left(k_{t}\left(w\left(A_{n}\right)\right)-k_{t}\left(A_{n}\right)\right) x, x\right\rangle_{0}\right| \leq C_{1} \eta \min \{1, t\}\|x\|_{0}^{2}, \quad x \in P_{n}\left(\mathcal{H}_{0}\right) .
$$

Now let $c>0$ be such that $A \geq c$. The elementary inequality $k_{t}(c) \geq(1 / 2) \min \{1, c t\}$ shows that

$$
\left\langle k_{t}\left(A_{n}\right) x, x\right\rangle_{0} \geq C_{2} \min \{1, t\}\|x\|_{0}^{2}, \quad x \in P_{n}\left(\mathcal{H}_{0}\right),
$$

where $C_{2}=(1 / 2) \min \{1, c\}$. Combining these estimates, we deduce that

$$
\left|\left\langle k_{t}\left(w\left(A_{n}\right)\right) x, x\right\rangle_{0}-\left\langle k_{t}\left(A_{n}\right) x, x\right\rangle_{0}\right| \leq C_{3} \eta\left\langle k_{t}\left(A_{n}\right) x, x\right\rangle_{0}, \quad x \in P_{n}\left(\mathcal{H}_{0}\right)
$$

for some suitable constant $C_{3}=C_{3}(n)$.

Now pick unit vectors $e_{i}, f_{i}$ supported by the spectral sets $E_{i} \cap \sigma\left(A_{n}\right)$ such that $x^{0}$ and $y^{0}$ belong to the space $\mathcal{W}$ spanned by $\left\{e_{i}, f_{i}\right\}_{1}^{N}$. Put $\mathcal{W}_{0}=\mathcal{W}_{1}=\mathcal{W}$ and define norms on those spaces by

$$
\|x\|_{\mathcal{W}_{0}}=\|x\|_{\mathcal{H}_{0}} \quad, \quad\|x\|_{\mathcal{W}_{1}}^{2}=\langle w(A) x, x\rangle_{\mathcal{H}_{0}} .
$$

The operator associated with $\overline{\mathcal{W}}$ is then the compression of $w\left(A_{n}\right)$ to $\mathcal{W}_{0}$, i.e.,

$$
\|x\|_{\mathcal{W}_{1}}^{2}=\left\langle A_{\overline{\mathcal{W}}} x, x\right\rangle_{\mathcal{W}_{0}}=\left\langle w\left(A_{n}\right) x, x\right\rangle_{\mathcal{H}_{0}}, \quad x \in \mathcal{W} .
$$

Let $\epsilon=2 C_{3} \eta$ and observe that, by (5.5)

$$
|K(t, x ; \overline{\mathcal{W}})-K(t, x ; \overline{\mathcal{H}})| \leq(\epsilon / 2) K(t, x ; \overline{\mathcal{H}}), \quad f \in \mathcal{W} .
$$


The eigenvalues of $A_{\overline{\mathcal{W}}}$ typically have multiplicity 2 . To obtain unit multiplicity, we perturb $A_{\overline{\mathcal{W}}}$ slightly to a positive matrix $A_{\overline{\mathcal{V}}}$ such that $\left\|A_{\overline{\mathcal{W}}}-A_{\overline{\mathcal{V}}}\right\|_{\mathcal{L}\left(\mathcal{H}_{0}\right)}<\epsilon / 2 C_{3}$. Let $\overline{\mathcal{V}}$ be the couple associated with $A_{\overline{\mathcal{V}}}$, i.e., put $\mathcal{V}_{i}=\mathcal{W}$ for $i=0,1$ and

$$
\|x\|_{\mathcal{V}_{0}}=\|x\|_{\mathcal{W}_{0}} \quad \text { and } \quad\|x\|_{\mathcal{W}_{1}}^{2}=\left\langle A_{\overline{\mathcal{V}}} x, x\right\rangle_{\mathcal{V}_{0}} .
$$

It is then straightforward to check that

$$
|K(t, f ; \overline{\mathcal{W}})-K(t, f ; \overline{\mathcal{V}})| \leq(\epsilon / 2) K(t, f ; \overline{\mathcal{H}}), \quad f \in \mathcal{W} .
$$

Combining this with the estimate (5.6), one finishes the proof of the lemma.

Proof of Theorem \. Given two elements $x^{0}, y^{0} \in \Sigma$ as in (5.2) we write $x^{n}=$ $P_{n}\left(x^{0}\right)$ and $y^{n}=P_{n}\left(y^{0}\right)$. By the proof of Lemma 5.2 we then have $K\left(t, y^{n}\right) \leq$ $\tilde{\rho}^{-1} K\left(t, x^{n}\right)$ for large enough $n$, where $\tilde{\rho}$ is any given number in the interval $(1, \rho)$.

We then use Lemma 5.3 to choose a finite-dimensional sub-couple $\overline{\mathcal{V}} \subset \overline{\mathcal{H}^{(n)}}$ such that

$$
\begin{aligned}
K\left(t, y^{n} ; \overline{\mathcal{V}}\right) & \leq(1+\epsilon) K\left(t, y^{n} ; \overline{\mathcal{H}}\right) \\
& <\tilde{\rho}^{-1} K\left(t, x^{n} ; \overline{\mathcal{V}}\right)+\epsilon\left(K\left(t, x^{n} ; \overline{\mathcal{H}}\right)+K\left(t, y^{n} ; \overline{\mathcal{H}}\right)\right)
\end{aligned}
$$

Here $\epsilon>0$ is at our disposal.

Choosing $\epsilon$ sufficiently small, we can arrange that

$$
K\left(t, y^{n} ; \overline{\mathcal{V}}\right) \leq K\left(t, x^{n} ; \overline{\mathcal{V}}\right), \quad t>0 .
$$

By Theorem 2.4 the condition (5.7) implies the existence of an operator $T^{\prime} \in$ $\mathcal{L}_{1}(\overline{\mathcal{V}})$ such that $T^{\prime} x^{n}=y^{n}$. Considering the canonical inclusion and projection

$$
I: \Sigma(\mathcal{V}) \rightarrow \Sigma(\mathcal{H}) \text { and } \Pi: \Sigma(\mathcal{H}) \rightarrow \Sigma(\mathcal{V}),
$$

we have, by virtue of Lemma 5.3 ,

$$
\|I\|_{\mathcal{L}(\overline{\mathcal{V}} ; \overline{\mathcal{H}})}^{2} \leq(1-\epsilon)^{-1} \quad \text { and } \quad\|\Pi\|_{\mathcal{L}(\overline{\mathcal{H}} ; \overline{\mathcal{V}})}^{2} \leq 1+\epsilon .
$$

Now let $T=T_{\varepsilon}:=I T^{\prime} \Pi \in \mathcal{L}\left(\overline{\mathcal{H}^{(n)}}\right)$. Then $\|T\|^{2} \leq \frac{1+\epsilon}{1-\epsilon}$ and $T x^{n}=y^{n}$. As $\epsilon \downarrow 0$ the operators $T_{\epsilon}$ will cluster at some point $T \in \mathcal{L}_{1}\left(\overline{\mathcal{H}^{(n)}}\right)$ such that $T x^{n}=y^{n}$ (cf. Lemma 5.11).

We have shown that $\overline{\mathcal{H}^{(n)}}$ has the $K$-property. In view of Lemma 5.2 , this implies that $\overline{\mathcal{H}}$ has the same property. The proof of Theorem $\Pi$ is therefore complete.

\section{REPRESENTATIONS OF INTERPOLATION FUNCTIONS}

6.1. Quadratic interpolation methods. Let us say that an interpolation method defined on regular Hilbert couples taking values in Hilbert spaces is a quadratic interpolation method. (Donoghue [13] used the same phrase in a somewhat wider sense, allowing the methods to be defined on non-regular Hilbert couples as well.)

If $F$ is an exact quadratic interpolation method, and $\overline{\mathcal{H}}$ a Hilbert couple, then by Donoghue's theorem III there exists a positive Radon measure $\varrho$ on $[0, \infty]$ such that $F(\overline{\mathcal{H}})=\mathcal{H}_{\varrho}$, where the latter space is defined by the familiar norm $\|x\|_{\varrho}^{2}=$ $\int_{[0, \infty]}\left(1+t^{-1}\right) K(t, x) d \varrho(t)$.

A priori, the measure $\varrho$ could depend not only on $F$ but also on the particular $\overline{\mathcal{H}}$. That $\varrho$ is independent of $\overline{\mathcal{H}}$ can be realized in the following way. Let $\overline{\mathcal{H}^{\prime}}$ be a regular Hilbert couple such that every positive rational number is an eigenvalue of 
the associated operator. Let $B^{\prime}$ be the operator associated with the exact quadratic interpolation space $F\left(\overline{\mathcal{H}^{\prime}}\right)$. There is then clearly a unique $P^{\prime}$-function $h$ on $\sigma\left(A^{\prime}\right)$ such that $B^{\prime}=h\left(A^{\prime}\right)$, viz. there is a unique positive Radon measure $\varrho$ on $[0, \infty]$ such that $F\left(\overline{\mathcal{H}^{\prime}}\right)=\mathcal{H}_{\rho}^{\prime}$ (see $\$ 1.2$ for the notation).

If $\overline{\mathcal{H}}$ is any regular Hilbert couple, we can form the direct sum $\overline{\mathcal{S}}=\overline{\mathcal{H}^{\prime}} \oplus \overline{\mathcal{H}}$. Denote by $\tilde{A}$ the corresponding operator and let $\tilde{B}=\tilde{h}(\tilde{A})$ be the operator corresponding to the exact quadratic interpolation space $F(\overline{\mathcal{S}})$. Then $\tilde{h}(\tilde{A})=\tilde{h}\left(A^{\prime}\right) \oplus \tilde{h}(A)=$ $h\left(A^{\prime}\right) \oplus \tilde{h}(A)$. This means that $\tilde{h}\left(A^{\prime}\right)=h\left(A^{\prime}\right)$, i.e. $\tilde{h}=h$. In particular, the operator $B$ corresponding to the exact interpolation space $F(\overline{\mathcal{H}})$ is equal to $h(A)$. We have shown that $F(\overline{\mathcal{H}})=\mathcal{H}_{\varrho}$. We emphasize our conclusion with the following theorem.

Theorem 6.1. There is a one-to-one correspondence $\varrho \mapsto F$ between positive Radon measures and exact quadratic interpolation methods.

We will shortly see that Theorem 6.1 is equivalent to the theorem of Foias and Lions [17. As we remarked above, a more general version of the theorem, admitting for non-regular Hilbert couples, is found in Donoghue's paper [13].

6.2. Interpolation type and reiteration. In this subsection, we prove some general facts concerning quadratic interpolation methods; we shall mostly follow Fan 15 .

Fix a function $h \in P^{\prime}$ of the form

$$
h(\lambda)=\int_{[0, \infty]} \frac{(1+t) \lambda}{1+t \lambda} d \varrho(t) .
$$

It will be convenient to write $\overline{\mathcal{H}}_{h}$ for the corresponding exact interpolation space $\mathcal{H}_{\varrho}$. Thus, we shall denote

$$
\|x\|_{h}^{2}=\langle h(A) x, x\rangle_{0}=\int_{[0, \infty]}\left(1+t^{-1}\right) K(t, x) d \varrho(t) .
$$

More generally, we shall use the same notation when $h$ is any quasi-concave function on $\mathbf{R}_{+}$; then $\overline{\mathcal{H}}_{h}$ is a quadratic interpolation space, but not necessarily exact.

Recall that, given a function $\mathbf{H}$ of one variable, we say that $\mathcal{H}_{*}$ is of type $\mathbf{H}$ with respect to $\overline{\mathcal{H}}$ if $\|T\|_{\mathcal{L}\left(\mathcal{H}_{i}\right)}^{2} \leq M_{i}$ implies $\|T\|_{\mathcal{L}\left(\mathcal{H}_{*}\right)}^{2} \leq M_{0} \mathbf{H}\left(M_{1} / M_{0}\right)$.

We shall say that a quasi-concave function $h$ on $\mathbf{R}_{+}$is of type $\mathbf{H}$ if $\overline{\mathcal{H}}_{h}$ is of type $\mathbf{H}$ relative to any regular Hilbert couple $\overline{\mathcal{H}}$. The following result somewhat generalizes Theorem 3.2. The class of functions of type $\mathbf{H}$ clearly forms a convex cone.

Theorem 6.2. Let $h$ be of type $\mathbf{H}$, where $(i) \mathbf{H}(1)=1$ and $\mathbf{H}(t) \leq \max \{1, t\}$, and (ii) $\mathbf{H}$ has left and right derivatives $\theta_{ \pm}=H^{\prime}(1 \pm)$ at the point 1 , where $\theta_{-} \leq \theta_{+}$. Then for any positive constant $c$,

$$
\min \left\{\lambda^{\theta_{-}}, \lambda^{\theta_{+}}\right\} \leq \frac{h(c \lambda)}{h(c)} \leq \max \left\{\lambda^{\theta_{-}}, \lambda^{\theta_{+}}\right\}, \quad \lambda \in \mathbf{R}_{+} .
$$

In particular, if $\mathbf{H}(t)$ is differentiable at $t=1$ and $\mathbf{H}^{\prime}(1)=\theta$, then $h(\lambda)=\lambda^{\theta}$, $\lambda \in \mathbf{R}_{+}$.

Proof. Replacing $A$ by $c A$, it is easy to see that if $h$ is of type $\mathbf{H}$, then so is $h_{c}(t)=h(c t) / h(c)$. Fix $\mu>0$ and consider the function $h_{0}(t)=h_{c}(\mu t) / h_{c}(\mu)$. By 
Theorem II we have $h_{0}(t) \leq \mathbf{H}(t)$ for all $t$. Furthermore $h_{0}(1)=\mathbf{H}(1)=1$ by (i). Since $h_{0}$ is differentiable, the assumption (ii) now gives $\theta_{-} \leq h_{0}^{\prime}(1) \leq \theta_{+}$, or

$$
\theta_{-} \leq \frac{\mu h_{c}^{\prime}(\mu)}{h_{c}(\mu)} \leq \theta_{+}
$$

Dividing through by $\mu$ and integrating over the interval $[1, \lambda]$, one now verifies the inequalities in (6.1).

The following result provides a partial converse to Theorem II

Theorem 6.3. ([15]) Let $h \in P^{\prime}$ and $\operatorname{set} \mathbf{H}(t)=\sup _{s>0} h(s t) / h(s)$. Then $h$ is of type $\mathbf{H}$.

Proof. Let $T \in \mathcal{L}(\overline{\mathcal{H}})$ be a non-zero operator; put $M_{j}=\|T\|_{\mathcal{L}\left(\mathcal{H}_{j}\right)}^{2}$ and $M=$ $M_{1} / M_{0}$. We then have (by Lemma 1.1)

$$
\begin{aligned}
\|T x\|_{h}^{2} & =\int_{[0, \infty]}\left(1+t^{-1}\right) K(t, T x) d \varrho(t) \\
& \leq M_{0} \int_{[0, \infty]}\left(1+t^{-1}\right) K(t M, x) d \varrho(t) \\
& =M_{0} \int_{[0, \infty]}\left\langle\frac{(1+t) M A}{1+t M A} x, x\right\rangle_{0} d \varrho(t) \\
& =M_{0}\langle h(M A) x, x\rangle_{0} .
\end{aligned}
$$

Letting $E$ be the spectral resolution of $A$, we have

$$
\langle h(M A) x, x\rangle_{0}=\int_{0}^{\infty} h(M \lambda) d\left\langle E_{\lambda} x, x\right\rangle_{0} .
$$

Since $h(M \lambda) / h(\lambda) \leq \mathbf{H}(M)$, we conclude that

$$
\|T x\|_{h}^{2} \leq M_{0} \mathbf{H}(M) \int_{0}^{\infty} h(\lambda) d\left\langle E_{\lambda} x, x\right\rangle_{0}=M_{0} \mathbf{H}(M)\|x\|_{h}^{2},
$$

which finishes the proof.

Given a function $h$ of a positive variable, we define a new function $\tilde{h}$ by

$$
\tilde{h}(s, t)=s h(t / s) .
$$

The following reiteration theorem is due to Fan.

Theorem 6.4. ([15]) Let $h, h_{0}, h_{1} \in P^{\prime}$, and $\varphi(\lambda)=\tilde{h}\left(h_{0}(\lambda), h_{1}(\lambda)\right)$. Then $\overline{\mathcal{H}}_{\varphi}=\left(\overline{\mathcal{H}}_{h_{0}}, \overline{\mathcal{H}}_{h_{1}}\right)_{h}$ with equal norms. Moreover, $\overline{\mathcal{H}}_{\varphi}$ is an exact interpolation space relative to $\overline{\mathcal{H}}$.

Proof. Let $\overline{\mathcal{H}^{\prime}}$ denote the couple $\left(\overline{\mathcal{H}}_{h_{0}}, \overline{\mathcal{H}}_{h_{1}}\right)$. The corresponding operator $A^{\prime}$ then obeys

$$
\|x\|_{\overline{\mathcal{H}}_{h_{1}}}=\left\|\left(A^{\prime}\right)^{1 / 2} x\right\|_{\mathcal{H}_{0}^{\prime}}=\left\|\varphi_{0}(A)^{1 / 2}\left(A^{\prime}\right)^{1 / 2} x\right\|_{0}, \quad x \in \Delta\left(\overline{\mathcal{H}^{\prime}}\right) .
$$

On the other hand, $\|x\|_{\overline{\mathcal{H}}_{h_{1}}}=\left\|\varphi_{1}(A)^{1 / 2} x\right\|_{0}$, so

$$
\left(A^{\prime}\right)^{1 / 2} x=\varphi_{0}(A)^{-1 / 2} \varphi_{1}(A)^{1 / 2} x, \quad x \in \Delta\left(\overline{\mathcal{H}^{\prime}}\right) .
$$


We have shown that $A^{\prime}=\varphi_{0}(A)^{-1} \varphi_{1}(A)$, whence (by Lemma 1.1)

$$
\begin{aligned}
K\left(t, x ; \overline{\mathcal{H}^{\prime}}\right) & =\left\langle\frac{t \varphi_{0}(A)^{-1} \varphi_{1}(A)}{1+t \varphi_{0}(A)^{-1} \varphi_{1}(A)} x, x\right\rangle_{\mathcal{H}_{0}^{\prime}} \\
& =\left\langle\frac{t \varphi_{1}(A)}{1+t \varphi_{0}(A)^{-1} \varphi_{1}(A)} x, x\right\rangle_{\mathcal{H}_{0}^{\prime}} .
\end{aligned}
$$

Now let the function $h \in P^{\prime}$ be given by

$$
h(\lambda)=\int_{[0, \infty]} \frac{(1+t) \lambda}{1+t \lambda} d \varrho(t),
$$

and note that the function $\varphi=\tilde{h}\left(h_{0}, h_{1}\right)$ is given by

$$
\varphi(\lambda)=\int_{[0, \infty]} \frac{(1+t) h_{1}(\lambda)}{1+t h_{1}(\lambda) / h_{0}(\lambda)} d \varrho(t) .
$$

Combining with (6.2), we find that

$$
\begin{aligned}
\|x\|_{\overline{\mathcal{H}}^{\prime} h}^{2} & =\int_{[0, \infty]}\left(1+t^{-1}\right) K\left(t, x ; \overline{\mathcal{H}^{\prime}}\right) d \varrho(t) \\
& =\int_{0}^{\infty}\left[\int_{[0, \infty]} \frac{(1+t) h_{1}(\lambda)}{1+t h_{1}(\lambda) / h_{0}(\lambda)} d \varrho(t)\right] d\left\langle E_{\lambda} x, x\right\rangle_{0}=\|x\|_{\mathcal{H}_{\varphi}}^{2} .
\end{aligned}
$$

This finishes the proof of the theorem.

Combining with Donoghue's theorem III one obtains the following, purely functiontheoretic corollary. Curiously, we are not aware of a proof which does not use interpolation theory.

Corollary 6.5. (15]) Suppose that $h \in P^{\prime}$ and that $h_{0}, h_{1} \in P^{\prime} \mid F$, where $F$ is some closed subset of $\mathbf{R}_{+}$. Then the function $\varphi=\tilde{h}\left(h_{0}, h_{1}\right)$ is also of class $P^{\prime} \mid F$.

6.3. Donoghue's representation. Let $\overline{\mathcal{H}}$ be a regular Hilbert couple. In Donoghue's setting, the principal object is the space $\Delta=\mathcal{H}_{0} \cap \mathcal{H}_{1}$ normed by $\|x\|_{\Delta}^{2}=$ $\|x\|_{0}^{2}+\|x\|_{1}^{2}$. In the following, all involutions are understood to be taken with respect to the norm of $\Delta$.

We express the norms in the spaces $\mathcal{H}_{i}$ as

$$
\|x\|_{0}^{2}=\langle H x, x\rangle_{\Delta} \quad \text { and } \quad\|x\|_{1}^{2}=\langle(1-H) x, x\rangle_{\Delta},
$$

where $H$ is a bounded positive operator on $\Delta, 0 \leq H \leq 1$. The regularity of $\overline{\mathcal{H}}$ means that neither 0 , nor 1 is an eigenvalue of $H$.

To an arbitrary quadratic intermediate space $\mathcal{H}_{*}$ there corresponds a bounded positive injective operator $K$ on $\Delta$ such that

$$
\|x\|_{*}^{2}=\langle K x, x\rangle_{\Delta} .
$$

It is then easy to see that $\mathcal{H}_{*}$ is exact interpolation if and only if, for bounded operators $T$ on $\Delta$, the conditions $T^{*} H T \leq H$ and $T^{*}(1-H) T \leq 1-H$ imply $T^{*} K T \leq K$. It is straightforward to check that the relations between $H, K$ and the operators $A, B$ used in the previous sections are:

$$
H=\frac{1}{1+A} \quad, \quad A=\frac{1-H}{H} \quad, \quad K=\frac{B}{1+A} \quad, \quad B=\frac{K}{H} .
$$

(It follows from the proof of Lemma 1.2 that $H$ and $K$ commute.) 
By Theorem 【II we know that $\mathcal{H}_{*}$ is an exact interpolation space if and only if $B=h(A)$ for some $h \in P^{\prime}$. By (6.3), this is equivalent to that $K=k(H)$ where

$$
k(H)=\frac{h(A)}{1+A}=H h\left(\frac{1-H}{H}\right) .
$$

In its turn, this means that

$$
\begin{aligned}
k(\lambda) & =\lambda \int_{[0, \infty]} \frac{(1+t)(1-\lambda) / \lambda}{1+t(1-\lambda) / \lambda} d \varrho(t) \\
& =\int_{[0, \infty]} \frac{(1+t) \lambda(1-\lambda)}{\lambda+t(1-\lambda)} d \varrho(t), \quad \lambda \in \sigma(H),
\end{aligned}
$$

where $\varrho$ is a suitable Radon measure. Applying the change of variables $s=1 /(1+t)$ and defining a positive Radon measure $\mu$ on $[0,1]$ by $d \mu(s)=d \varrho(t)$, we arrive at the expression

$$
k(\lambda)=\int_{0}^{1} \frac{\lambda(1-\lambda)}{(1-s)(1-\lambda)+s \lambda} d \mu(s), \quad \lambda \in \sigma(H),
$$

which gives the representation exact quadratic interpolation spaces originally used by Donoghue in [13].

6.4. $J$-methods and the Foiaş-Lions theorem. We define the (quadratic) $J$ functional relative to a regular Hilbert couple $\overline{\mathcal{H}}$ by

$$
J(t, x)=J(t, x ; \overline{\mathcal{H}})=\|x\|_{0}^{2}+t\|x\|_{1}^{2}, \quad t>0, x \in \Delta(\overline{\mathcal{H}}) .
$$

Note that $J(t, x)^{1 / 2}$ is an equivalent norm on $\Delta$ and that $J(1, x)=\|x\|_{\Delta}^{2}$.

Given a positive Radon measure $\nu$ on $[0, \infty]$, we define a Hilbert space $J_{\nu}(\overline{\mathcal{H}})$ as the set of all elements $x \in \Sigma(\overline{\mathcal{H}})$ such that there exists a measurable function $u:[0, \infty] \rightarrow \Delta$ such that

$$
x=\int_{[0, \infty]} u(t) d \nu(t) \quad(\text { convergence in } \Sigma)
$$

and

$$
\int_{[0, \infty]} \frac{J(t, u(t))}{1+t} d \nu(t)<\infty .
$$

The norm in the space $J_{\nu}(\overline{\mathcal{H}})$ is defined by

$$
\|x\|_{J_{\nu}}^{2}=\inf _{u} \int_{[0, \infty]} \frac{J(t, u(t))}{1+t} d \nu(t)
$$

over all $u$ satisfying (6.5) and (6.6).

The space (6.7) was (with different notation) introduced by Foiaş and Lions in the paper [17, where it was shown that there is a unique minimizer $u(t)$ of the problem (6.7), namely

$$
u(t)=\varphi_{t}(A) x \quad \text { where } \quad \varphi_{t}(\lambda)=\frac{1+t}{1+t \lambda}\left(\int_{[0, \infty]} \frac{1+s}{1+s \lambda} d \nu(s)\right)^{-1} .
$$

Inserting this expression for $u$ into (6.7), one finds that

$$
\|x\|_{J_{\nu}}^{2}=\langle h(A) x, x\rangle_{0}
$$


where

$$
h(\lambda)^{-1}=\int_{[0, \infty]} \frac{1+t}{1+t \lambda} d \nu(t) .
$$

It is easy to verify that the class of functions representable in the form (6.9) for some positive Radon measure $\nu$ coincides with the class $P^{\prime}$. We have thus arrived at the following result.

Theorem 6.6. Every exact quadratic interpolation space $\mathcal{H}_{*}$ can be represented isometrically in the form $\mathcal{H}_{*}=J_{\nu}(\overline{\mathcal{H}})$ for some positive Radon measure $\nu$ on $[0, \infty]$. Conversely, any space of this form is an exact quadratic interpolation space.

In the original paper [17, Foias and Lions proved the less precise statement that each exact quadratic interpolation method $F$ can be represented as $F=J_{\nu}$ for some positive Radon measure $\nu$.

6.5. The relation between the $K$ - and $J$-representations. The assignment $K_{\varrho}=J_{\nu}$ gives rise to a non-trivial bijection $\varrho \mapsto \nu$ of the set of positive Radon measures on $[0, \infty]$. In this bijection, $\varrho$ and $\nu$ are in correspondence if and only if

$$
\int_{[0, \infty]} \frac{(1+t) \lambda}{1+t \lambda} d \varrho(t)=\left(\int_{[0, \infty]} \frac{1+t}{1+t \lambda} d \nu(t)\right)^{-1} .
$$

As an example, let us consider the geometric interpolation space (where $c_{\theta}=$ $\pi / \sin (\pi \theta))$

$$
\|x\|_{\theta}^{2}=\left\langle A^{\theta} x, x\right\rangle_{0}=c_{\theta} \int_{0}^{\infty} t^{-\theta} K(t, x) \frac{d t}{t} .
$$

The measure $\varrho$ corresponding to this method is $d \varrho_{\theta}(t)=\frac{c_{\theta} t^{-\theta}}{1+t} d t$. On the other hand, it is easy to check that

$$
\lambda^{\theta}=\left(\int_{0}^{\infty} \frac{1+t}{1+t \lambda} d \nu_{\theta}(t)\right)^{-1} \quad \text { where } \quad d \nu_{\theta}(t)=\frac{c_{\theta} t^{\theta}}{1+t} \frac{d t}{t} .
$$

We leave it to the reader to check that the norm in $\mathcal{H}_{\theta}$ is the infimum of the expression

$$
c_{\theta} \int_{0}^{\infty} t^{\theta} J(t, u(t)) \frac{d t}{t}
$$

over all representations

$$
x=\int_{0}^{\infty} u(t) \frac{d t}{t} .
$$

We have arrived at the Hilbert space version of Peetre's $J$-method of exponent $\theta$. The identity $J_{\nu_{\theta}}=K_{\varrho_{\theta}}$ can now be recognized as a sharp (isometric) Hilbert space version of the equivalence theorem of Peetre, which says that the standard $K_{\theta}$ and $J_{\theta}$-methods give rise to equivalent norms on the category of Banach couples (see [7]).

The problem of determining the pairs $\varrho, \nu$ having the property that the $K_{\varrho}$ and $J_{\nu}$ methods give equivalent norms was studied by Fan in [15, Section 3]. 
6.6. Other representations. As we have seen in the preceding subsections, using the space $\mathcal{H}_{0}$ to express all involutions and inner products leads to a description of the exact quadratic interpolation spaces in terms of the class $P^{\prime}$. If we instead use the space $\Delta$ as the basic object, we get Donoghue's representation for interpolation functions. Similarly, one can proceed from any fixed interpolation space $\mathcal{H}_{*}$ to obtain a different representation of interpolation functions.

6.7. On interpolation methods of power $p$. Fix a number $p, 1<p<\infty$. We shall write $L_{p}=L_{p}(X, \mathcal{A}, \mu)$ for the usual $L_{p}$-space associated with an arbitrary but fixed ( $\sigma$-finite) measure $\mu$ on a measure space $(X, \mathcal{A})$. Given a positive measurable weight function $w$, we write $L_{p}(w)$ for the space normed by

$$
\|f\|_{L_{p}(w)}^{p}=\int_{X}|f(x)|^{p} w(x) d \mu(x) .
$$

We shall write $\bar{L}_{p}(w)=\left(L_{p}, L_{p}(w)\right)$ for the corresponding weighted $L_{p}$ couple. Note that the conditions imposed mean precisely that $\bar{L}_{p}(w)$ be separable and regular.

Let us say that an exact interpolation functor $F$ defined on the totality of separable, regular weighted $L_{p}$-couples and taking values in the class of weighted $L_{p}$-spaces is of power $p$.

Define, for a positive Radon measure $\varrho$ on $[0, \infty]$, an exact interpolation functor $F=K_{\varrho}(p)$ by the definition

$$
\|f\|_{F\left(\bar{L}_{p}(w)\right)}^{p}:=\int_{[0, \infty]}\left(1+t^{-\frac{1}{p-1}}\right)^{p-1} K_{p}\left(t, f ; \bar{L}_{p}(w)\right) d \varrho(t) .
$$

We contend that $F$ is of power $p$.

Indeed, it is easy to verify that

$$
K_{p}\left(t, f ; \bar{L}_{p}(w)\right)=\int_{X}|f(x)|^{p} \frac{t w(x)}{\left(1+(t w(x))^{\frac{1}{p-1}}\right)^{p-1}} d \mu(x),
$$

so Fubini's theorem gives that

$$
\|f\|_{F\left(\bar{L}_{p}(w)\right)}^{p}=\int_{X}|f(x)|^{p} h(w(x)) d \mu(x),
$$

where

$$
h(\lambda)=\int_{[0, \infty]} \frac{\left(1+t^{\frac{1}{p-1}}\right)^{p-1} \lambda}{\left(1+(t \lambda)^{\frac{1}{p-1}}\right)^{p-1}} d \varrho(t), \quad \lambda \in w(X) .
$$

We have shown that $F\left(\bar{L}_{p}(w)\right)=L_{p}(h(w))$, so $F$ is indeed of power $p$.

Let us denote by $\mathcal{K}(p)$ the totality of positive functions $h$ on $\mathbf{R}_{+}$representable in the form (6.10) for some positive Radon measure $\varrho$ on $[0, \infty]$.

Further, let $\mathcal{I}(p)$ denote the class of all (exact) interpolation functions of power $p$, i.e., those positive functions $h$ on $\mathbf{R}_{+}$having the property that for each weighted $L_{p}$ couple $\bar{L}_{p}(w)$ and each bounded operator $T$ on $\bar{L}_{p}(w)$, it holds that $T$ is bounded on $L_{p}(h(w))$ and

$$
\|T\|_{\mathcal{L}\left(L_{p}(h(w))\right)} \leq\|T\|_{\mathcal{L}\left(\bar{L}_{p}(w)\right)} .
$$

The class $\mathcal{I}(p)$ is in a sense the natural candidate for the class of "operator monotone functions on $L_{p}$-spaces". The class $\mathcal{I}(p)$ clearly forms a convex cone; it was shown by Peetre [31] that this cone is contained in the class of concave positive functions on $\mathbf{R}_{+}$(with equality if $p=1$ ). 
We have shown that $\mathcal{K}(p) \subset \mathcal{I}(p)$. By Theorem 6.1. we know that equality holds when $p=2$. For other values of $p$ it does not seem to be known whether the class $\mathcal{K}(p)$ exhausts the class $\mathcal{I}(p)$, but one can show that we would have $\mathcal{K}(p)=\mathcal{I}(p)$ provided that each finite-dimensional $L_{p^{-}}$couple $\overline{\ell_{p}^{n}}(\lambda)$ has the $K_{p^{-}}$ property (or equivalently, the $K$-property, see (2.2)). Naturally, the latter problem (about the $K_{p}$-property) also seems to be open, but some comments on it are found in Remark 2.9.

Let $\nu$ be a positive Radon measure on $[0, \infty]$. In [17], Foiaş and Lions introduced a method, which we will denote by $F=J_{\nu}(p)$ in the following way. Define the $J_{p^{-}}$ functional by

$$
J_{p}\left(t, f ; \bar{L}_{p}(\lambda)\right)=\|f\|_{0}^{p}+t\|f\|_{1}^{p}, \quad f \in \Delta, t>0 .
$$

We then define an intermediate norm by

$$
\|f\|_{F\left(\bar{L}_{p}(\lambda)\right)}^{p}:=\inf \int_{[0, \infty]}(1+t)^{-\frac{1}{p-1}} J_{p}\left(t, u(t) ; \bar{L}_{p}(\lambda)\right) d \nu(t),
$$

where the infimum is taken over all representations

$$
f=\int_{[0, \infty]} u(t) d \nu(t)
$$

with convergence in $\Sigma$. It is straightforward to see that the method $F$ so defined is exact; in [17] it is moreover shown that it is of power $p$. More precisely, it is there proved that

$$
\|f\|_{F\left(\bar{L}_{p}(\lambda)\right)}^{p}=\int_{X}|f(x)|^{p} h(w(x)) d \mu(x),
$$

where

$$
h(\lambda)^{-\frac{1}{p-1}}=\int_{[0, \infty]} \frac{(1+t)^{\frac{1}{p-1}}}{(1+t \lambda)^{\frac{1}{p-1}}} d \nu(t), \quad \lambda \in w(X) .
$$

Let us denote by $\mathcal{J}(p)$ the totality of functions $h$ representable in the form (6.11). We thus have that $\mathcal{J}(p) \subset \mathcal{I}(p)$. In view of our preceding remarks, we conclude that if all weighted $L_{p}$-couples have the $K_{p}$ property, then necessarily $\mathcal{J}(p) \subset \mathcal{K}(p)$. Note that $\mathcal{J}(2)=\mathcal{K}(2)$ by Theorem 6.6 .

\section{Appendix: The COMPlex Method is QUADRATIC}

Let $S=\{z \in \mathbf{C} ; 0 \leq \operatorname{Re} z \leq 1\}$. Fix a Hilbert couple $\overline{\mathcal{H}}$ and let $\mathcal{F}$ be the set of functions $S \rightarrow \Sigma$ which are bounded and continuous in $S$, analytic in the interior of $S$, and which maps the line $j+i \mathbf{R}$ into $\mathcal{H}_{j}$ for $j=0,1$. Fix $0<\theta<1$. The norm in the complex interpolation space $C_{\theta}(\overline{\mathcal{H}})$ is defined by

$$
\|x\|_{C_{\theta}(\overline{\mathcal{H}})}=\inf \left\{\|f\|_{\mathcal{F}} ; f(\theta)=x\right\} .
$$

Let $\mathcal{P}$ denote the set of polynomials $f=\sum_{1}^{N} a_{i} z^{i}$ where $a_{i} \in \Delta$. We endow $\mathcal{P}$ with the inner product

$$
\langle f, g\rangle_{M_{\theta}}=\sum_{j=0,1} \int_{\mathbf{R}}\langle f(j+i t), g(j+i t)\rangle_{j} P_{j}(\theta, t) d t,
$$

where $\left\{P_{0}, P_{1}\right\}$ is the Poisson kernel for $S$,

$$
P_{j}(\theta, t)=\frac{e^{-\pi t} \sin \theta \pi}{\sin ^{2} \theta \pi+\left(\cos \theta \pi-(-1)^{j} e^{-\pi t}\right)^{2}} .
$$


Let $M_{\theta}$ be the completion of $\mathcal{P}$ with this inner product. It is easy to see that the elements of $M_{\theta}$ are analytic in the interior of $S$, and that evaluation map $f \mapsto f(\theta)$ is continuous on $M_{\theta}$. Let $N_{\theta}$ be the kernel of this functional and define a Hilbert space $\mathcal{H}_{\theta}$ by

$$
\mathcal{H}_{\theta}=M_{\theta} / N_{\theta}
$$

We denote the norm in $\mathcal{H}_{\theta}$ by $\|\cdot\|_{\theta}$.

Proposition A.1. $C_{\theta}(\overline{\mathcal{H}})=\mathcal{H}_{\theta}$ with equality of norms.

Proof. Let $f \in \mathcal{F}$. By the Calderón lemma in [7, Lemma 4.3.2], we have the estimate

$$
\log \|f(\theta)\|_{C_{\theta}(\overline{\mathcal{H}})} \leq \sum_{j=0,1} \int_{\mathbf{R}} \log \|f(j+i t)\|_{j} P_{j}(\theta, t) d t .
$$

Applying Jensen's inequality, this gives that

$$
\|f(\theta)\|_{C_{\theta}(\overline{\mathcal{H}})} \leq\left(\sum_{j=0,1} \int_{\mathbf{R}}\|f(j+i t)\|_{j}^{2} P_{j}(\theta, t) d t\right)^{1 / 2}=\|f\|_{M_{\theta}} .
$$

Hence $\mathcal{H}_{\theta} \subset C_{\theta}(\overline{\mathcal{H}})$ and $\|\cdot\|_{C_{\theta}(\overline{\mathcal{H}})} \leq\|\cdot\|_{\theta}$. On the other hand, for $f \in \mathcal{P}$ one has the estimates

$$
\|f(\theta)\|_{\theta} \leq\|f\|_{M_{\theta}} \leq \sup \left\{\|f(j+i t)\|_{j} ; t \in \mathbf{R}, j=0,1\right\}=\|f\|_{\mathcal{F}},
$$

whence $C_{\theta}(\overline{\mathcal{H}}) \subset \mathcal{H}_{\theta}$ and $\|\cdot\|_{C_{\theta}(\overline{\mathcal{H}})} \geq\|\cdot\|_{\theta}$.

It is well known that the method $C_{\theta}$ is of exponent $\theta$ (see e.g. [7]). We have shown that $C_{\theta}$ is an exact quadratic interpolation method of exponent $\theta$.

Complex interpolation with derivatives. In [15, pp. 421-422], Fan considers the more general complex interpolation method $C_{\theta(n)}$ for the $n$ :th derivative. This means that in $\left(^{*}\right)$, one consider representations $x=\frac{1}{n !} f^{(n)}(\theta)$ where $f \in \mathcal{F}$; the complex method $C_{\theta}$ is thus the special case $C_{\theta(0)}$. It is shown in 15 that, for $n \geq 1$, the $C_{\theta(n)}$-method is represented, up to equivalence of norms, by the quasi-power function $h(\lambda)=\lambda^{\theta} /\left(1+\frac{\theta(1-\theta)}{n}|\log \lambda|\right)^{n}$. The complex method with derivatives was introduced by Schechter [37]; for more details on that method, we refer to the list of references in [15.

\section{REFERENCES}

[1] Agler, J., McCarthy, J. E., Young, N., Operator monotone functions and Löwner functions of several variables, Ann. Math. 176 (2012), 1783-1826.

[2] Ameur, Y., The Calderón problem for Hilbert couples, Ark. Mat. 41 (2003), 203-231.

[3] Ameur, Y., A new proof of Donoghue's interpolation theorem, Journal of Function Spaces and Applications 3 (2004), 253-265.

[4] Ameur, Y., A note on a theorem of Sparr, Math. Scand. 94 (2004), 155-160.

[5] Ameur, Y., Cwikel, M., On the K-divisibility constant for some special, finite-dimensional Banach couples, J. Math. Anal. Appl. 360 (2009), 130-155.

[6] Aronszajn, N., Donoghue, W., On exponential representations of functions, J. Analyse Math. 5 (1956-57), 321-388.

[7] Bergh, J., Löfström, J., Interpolation spaces, an introduction. Springer 1976.

[8] Brudnyi, Y. A., Krugljak, N. Y., Interpolation functors and interpolation spaces, North Holland 1991.

[9] Calderón, A. P., Intermediate spaces and interpolation, the complex method, Studia Math. 24 (1964), 113-190. 
[10] Calderón, A. P., Spaces between $L_{1}$ and $L_{\infty}$ and the theorem of Marcinkiewicz, Studia Math. 26 (1966), 273-299.

[11] Cwikel, M., Monotonicity properties of interpolation spaces, Ark. Mat. 14 (1976), 213-236.

[12] Donoghue, W., Monotone matrix functions and analytic continuation, Springer 1974.

[13] Donoghue, W., The interpolation of quadratic norms, Acta Math. 118 (1967), 251-270.

[14] Donoghue, W., The theorems of Loewner and Pick, Israel J. Math. 4 (1966), 153-170.

[15] Fan, M., Quadratic interpolation and some operator inequalities, Journal of Mathematical Inequalities 5 (2011), 413-427.

[16] Foiaş, C., Ong, S. C., Rosenthal, P., An interpolation theorem and operator ranges, Integral Equations Operator Theory 10 (1987), 802-811.

[17] Foiaş, C., Lions, J. L., Sur certains théorèmes d'interpolation, Acta Sci. Math. 22 (1961), 269-282.

[18] Halmos, P. R., Quadratic interpolation, J. Operator Theory 7 (1982), 303-305.

[19] Hansen, F., An operator inequality, Math. Ann. 246 (1980), 249-250.

[20] Hansen, F., Selfadjoint means and operator monotone functions, Math. Ann. 256 (1981), $29-35$.

[21] Heinävaara, O., Local characterizations for the matrix monotonicity and convexity of fixed order, Proc. Amer. math. Soc. 146 (2018), 3791-3799.

[22] Korányi, A., On some classes of analytic functions of several variables, Trans. Amer. Math. Soc. 101 (1961), 520-554.

[23] Kraus, F., Über konvexe Matrixfunktionen, Math. Z. 41 (1936), 18-42.

[24] Lions, J. L., Espaces intermédiaires entre espaces Hilbertiens et applications, Bull. Math. de la Soc. Sci. Math. Phys. de la R. P. R. 2 (1958), 419-432.

[25] Lions, J. L., Magenes, E., Non-homogeneous boundary value problems and applications 1, Springer 1972.

[26] Löwner, K., Über monotone Matrixfunktionen, Math. Z. 38 (1934), 177-216.

[27] McCarthy, J. E., Geometric interpolation of Hilbert spaces, Ark. Mat. 30 (1992), 321-330.

[28] Mityagin, B., An interpolation theorem for modular spaces, Mat. Sbornik 66 (1965), 473482.

[29] Murphy, G. J., C $C^{*}$-algebras and operator theory, Academic Press 1991.

[30] Ovchinnikov, V. I., The method of orbits in interpolation theory, Math. Rep. 1 (1984), 349-515.

[31] Peetre, J., On interpolation functions III, Acta Szeged 30 (1969), 235-239.

[32] Peetre, J., Two new interpolation methods based on the duality map, Acta Math. 173 (1979), 73-91.

[33] Pusz, W., Woronowicz, S. L., Functional calculus for sesquilinear forms and the purification map, Rep. Math. Phys. 8 (1975), 159-170.

[34] Rosenblum, M., Rovnyak, J., Hardy classes and operator theory, Dover 1997.

[35] Sedaev, A., Description of interpolation spaces for the pair $\left(L_{p}\left(a_{0}\right), L_{p}\left(a_{1}\right)\right)$, Soviet Math. Dokl. 14 (1973), 538-541.

[36] Sedaev, A., Semenov, E. M., On the possibility of describing interpolation spaces in terms of the K-functional of Peetre, Optimizacja 4 (1971), 98-114.

[37] Shechter, M., Complex interpolation, Compositio Math. 18 (1967), 117-147.

[38] Sparr, G., A new proof of Löwner's theorem, Math. Scand 47 (1980), 266-274.

[39] Sparr, G., Interpolation of weighted $L^{p}$ spaces, Studia Math. 62 (1978), 229-271.

[40] Uhlmann, A., Relative entropy and the Wigner-Yanase-Dyson-Lieb concavity in an interpolation theory, Commun. Math. Phys. 54 (1977), 21-32.

[41] Vasudeva, H., On monotone matrix functions of two variables, Trans. Amer. Math. Soc. 176 (1973), 305-318.

Department of Mathematics, Faculty of Science, Lund University, P.O. Box 118, 22100 Lund, Sweden

E-mail address: Yacin.Ameur@math.lu.se 